\title{
Elucidation of global and local genomic epidemiology of Salmonella enterica serovar Enteritidis through multilevel genome typing
}

Lijuan Luo ${ }^{1}$, Michael Payne ${ }^{1}$, Sandeep Kaur ${ }^{1}$, Dalong $\mathrm{Hu}^{1}$, Liam Cheney ${ }^{1}$, Sophie Octavia ${ }^{1}$, Qinning Wang $^{2}$, Mark M. Tanaka ${ }^{1}$, Vitali Sintchenko ${ }^{2,3}$ and Ruiting Lan ${ }^{1, *}$

${ }^{1}$ School of Biotechnology and Biomolecular Sciences, University of New South Wales, Sydney, New South Wales, Australia

${ }^{2}$ Centre for Infectious Diseases and Microbiology-Public Health, Institute of Clinical Pathology and Medical Research - NSW Health Pathology, Westmead Hospital, New South Wales, Australia

${ }^{3}$ Marie Bashir Institute for Infectious Diseases and Biosecurity, Sydney Medical School, University of Sydney, New South Wales, Australia

*Corresponding Author

Email: r.lan@unsw.edu.au

Phone: 61-2-9385 2095

Fax: 61-2-9385 1483

1 Keywords: Salmonella enterica serovar Enteritidis; MGT; genomic epidemiology; population 2 structure; global database; virulence

4 Repositories: There were no newly sequenced data in this study. 


\section{Abstract}

7 Salmonella enterica serovar Enteritidis is a major cause of foodborne Salmonella infections and

8 outbreaks in humans. Effective surveillance and timely outbreak detection are essential for public

9 health control. Multilevel genome typing (MGT) with multiple levels of resolution has been

10 previously demonstrated as a promising tool for this purpose. In this study, we developed MGT with

11 nine levels for $S$. Enteritidis and characterised the genomic epidemiology of $S$. Enteritidis in detail.

12 We examined 26,670 publicly available $S$. Enteritidis genome sequences from isolates spanning 101

13 years from 86 countries to reveal their spatial and temporal distributions. Using the lower resolution

14 MGT levels, globally prevalent and regionally restricted sequence types (STs) were identified; avian associated MGT4-STs were found that were common in human cases in the USA were identified; temporal trends were observed in the UK with MGT5-STs from 2014 to 2018, revealing both long lived endemic STs and the rapid expansion of new STs. Using MGT3 to MGT6, we identified MDR associated STs at various MGT levels, which improves precision of detection and global tracking of MDR clones. We also found that the majority of the global $S$. Enteritidis population fell within two predominant lineages, which had significantly different propensity of causing large scale outbreaks. An online open MGT database has been established for unified international surveillance of $S$. Enteritidis. We demonstrated that MGT provides a flexible and high-resolution genome typing tool for $S$. Enteritidis surveillance and outbreak detection.

\section{Impact statement}

Salmonella enterica serovar Enteritidis is a common foodborne pathogen that can cause large outbreaks. Surveillance and high-resolution typing are essential for outbreak prevention and control.

Genome sequencing offers unprecedented power for these purposes and a standardised method or platform for the interpretation, comparison and communication of genomic typing data is highly desirable. In this work, we developed a genomic typing scheme called Multilevel Genome Typing (MGT) for $S$. Enteritidis. We analysed 26,670 publicly available genomes of $S$. Enteritidis using 
32 MGT. We characterised the geographic and temporal distribution of S. Enteritidis MGT types as well

33 as their association with multidrug resistance (MDR) and virulence genes. A publicly available MGT

34 database for $S$. Enteritidis was established, which has the potential facilitate the unified global public

35 health surveillance for this pathogen.

37 Abbreviations

38 MGT: Multilevel genome typing; ST: Sequence type; CC: Clonal complex; ODC: Outbreak 39 detection cluster; SNP: Single nucleotide polymorphism; MLST: Multi-locus sequence typing; 40 cgMLST: core genome multi-locus sequence typing; HierCC: Hierarchical clustering of cgMLST.

41 AR: Antibiotic resistance; MDR: Multi-drug resistance.

42

43 Data Summary

44 1. The MGT database for $S$. Enteritidis is available at https://mgtdb.unsw.edu.au/enteritidis/.

45 2. All accession numbers of the public available genomes were available in the MGT database and

46 Data Set S1, Tab 1. And there were no newly sequenced data in this study.

47 3. Supplementary material: Supplementary Fig. S1 to S7, supplementary methods and supporting 48 results about the evaluation of potential repeat sequencing bias.

49 4. Data Set S1: Supporting tables of the main results.

50 5. Data Set S2. Supporting tables of the repeat sequencing bias evaluation by removing the potential 51 repeat sequencing isolates. Note outbreak isolates may also be removed.

\section{Introduction}

53 Nontyphoidal Salmonella is ranked second in foodborne disease in Europe and North America [1, 2],

54 with Salmonella enterica serovar Enteritidis a dominant serovar in many countries [3, 4]. S.

55 Enteritidis mainly causes human gastrointestinal infections leading to diarrhoea. However, invasive 
56 infections manifesting as sepsis, meningitis and pneumonia, have recently been reported [5, 6]. As

57 many $S$. Enteritidis strains causing invasive infections carry plasmids which confer multidrug

58 resistance (MDR), the spread of these strains internationally is a major threat to global health $[7,8]$.

59 Additionally, S. Enteritidis has caused numerous large-scale national and international outbreaks

60 with complex transmission pathways $[3,9,10]$. While there have been limited studies of the total

61 global epidemiology of $S$. Enteritidis genomic subtypes [5, 11], the geographic distribution, outbreak

62 propensity, MDR and evolutionary characteristics of different lineages of $S$. Enteritidis have not been

63 systematically evaluated using the large numbers of newly available public genome sequences. A

64 rapid, stable and standardized global genomic typing strategy for $S$. Enteritidis is required for the

65 high-resolution and scalable surveillance of outbreaks, tracking of international spread and MDR 66 profiles of $S$. Enteritidis.

67 Sequence types (STs) are based on exact matching of genes between isolates. The well-established seven gene multi-locus sequence typing (MLST) of Salmonella is widely used [12], while the vast majority of $S$. Enteritidis are assigned to ST11, as only seven housekeeping genes are compared. STs of higher resolution are required. With this in mind core genome MLST (cgMLST) and whole genome MLST (wgMLST) schemes of Salmonella were developed including 3002 and 21,065 loci, respectively [13]. However, STs based on cgMLST and wgMLST are too diverse to offer useful relatedness information at anything but the finest scales. This issue was addressed by single linkage hierarchic typing systems, i.e., Single Nucleotide Polymorphism (SNP) address and Hierarchical Clustering of cgMLST (HierCC) [14-16]. SNP address is based on the single linkage hierarchic clustering method, which allows for different SNP differences 250, 100, 50, 25, 10, 5 and 0 SNPs [15]. These methods had been successfully used in outbreak tracing and epidemiology [14, 17-19]. However, one major disadvantage of the pairwise comparison based single linkage clustering method is that the cluster types called may merge when additional data is added and differences in order of addition can result in different clusters [20]. For example, when an isolate meets the SNP/allele 
81 difference threshold to two clusters, this isolate would act as a bridge connecting the two clusters and

82 merging them into a single cluster. This scenario will almost certainly occur in a large database with

83 ever expanding numbers of sequences, especially at finer resolutions, and would be an obstacle for

84 long term epidemiological investigation and comparison [20]. We have recently developed a novel

85 core genome based method called multilevel genome typing (MGT), an exact matching method to

86 assign multiple resolutions of relatedness without the need for clustering [20]. MGT is a genome

87 sequence based typing system, including multiple MLST schemes with increasing resolution from

88 the classic seven gene MLST to cgMLST where each scheme is used to independently assign an ST.

89 An MGT scheme and a public database were established for Salmonella enterica serovar

90 Typhimurium where seven to 5268 loci were included in MGT1 to 9 [20]. MGT1 corresponds to the

91 classic seven gene MLST scheme of S. enterica [12]. For S. Typhimurium, the stable STs from MGT

92 levels of appropriate resolution were found to be useful in identifying DT104, the MDR lineage, as

93 well as African invasive lineages [20]. MGT of $S$. Typhimurium was also shown to perform well in

94 outbreak identification and source tracing [20].

95 Here, we describe an MGT scheme and public database for $S$. Enteritidis. By using MGT, we 96 systematically examine the global and local epidemiology of $S$. Enteritidis over time, and evaluate 97 the application of this scheme to the outbreak evaluation and the monitoring of multidrug resistant 98 STs.

Methods

\section{$S$. Enteritidis sequences used in this study}

101 Raw reads of 26,670 S. Enteritidis whole genome sequences (WGS) downloaded from the European 102 Nucleotide Archive (ENA) passed the quality filtering criteria by Quast [21, 22] (Data Set S1, Tab 103 1). The epidemiological metadata of those isolates were collated from NCBI and EnteroBase [13]. 104 Trimmomatic v0.39 was used with default settings to trim raw reads [23]. The trimmed raw reads 
105 were then assembled using either SPAdes v3.13.0 for core genome definition or SKESA v2.3 for

106 MGT typing [24, 25]. Quality assessment for the assemblies were performed using Quast v5.0.2

107 [21]. Thresholds of the assembly quality were in accordance with Robertson et al 's criteria [22].

108 Kraken v0.10.5 was used to identify contamination in the assembled genomic sequences or 109 problematic taxonomic identification [26]. Seqsero v1.0 and SISTR v1.0.2 were used to verify 110 serotype assignments [27, 28].

\section{Core gene definition of $S$. Enteritidis}

112 To define the core genome of $S$. Enteritidis, a total of 1801 genomes were selected based on the 113 ribosomal sequence type [13] and strain metadata. The trimmed raw reads were then assembled 114 using SPAdes v3.13.0 [24]. The assemblies were annotated with Prokka v1.12[29]. The core genes 115 and core intergenic regions were defined with Roary v3.11.2 and Piggy v3.11.2, respectively (Fig. 116 S1). A total of 113 sRNA regions of SL1344 were searched in the 1054 intergenic regions using 117 BLAST, with identity and coverage threshold of $70 \%$ and $60 \%$, respectively [30]. Both SeqSero v1.0 118 and SISTR v1.0.2 were used to verify whether the isolates were S. Enteritidis [27, 28]. The detailed 119 methods for isolates selection and core genome identification were described in Supplementary 120 material.

\section{Establishment of MGT scheme for $S$. Enteritidis and MGT allele calling}

122 The initial allele sequence of each locus used for MGT were extracted from the complete genome 123 sequence of $S$. Enteritidis strain P125109 (Genbank accession NC_011294.1). The first eight levels 124 of MGT were identical to the MGT scheme for $S$. Typhimurium [20], in which MGT1 refers to the 125 classical seven gene MLST for the Salmonella genus [12]. Loci used for levels MGT2 to MGT8 126 were orthologous to those in the $S$. Typhimurium MGT with the exception of one gene which was 127 removed at MGT8 because of a paralogue in $S$. Enteritidis. MGT9 contains all loci in MGT8 as well 128 as the core genes and core intergenic regions of $S$. Enteritidis. 
129 The raw read processing and genome assembly procedures were the same as above, except that 130 SKESA v2.3 was used to assemble the raw reads and only SISTR v1.0.2 was used for the molecular 131 serotype identification [25, 28]. SKESA v2.3 offers higher per base accuracy and speed than 132 SPADES and was therefore used in the allele calling pipeline [25]. For the assemblies that passed 133 quality control by the criteria of Robertson et al. [22], a custom script was used to assign alleles, STs 134 and clonal complexes (CCs) to those isolates at levels from MGT2 to MGT9 [20]. Allele, ST, CC 135 and outbreak detection cluster (ODC) assignments were performed as previously described [20]. 136 Briefly, if one or more allele difference was observed, a new ST was assigned to the isolate. The 137 same strategy was also used for assigning a new CC, if at least two allele differences were observed 138 comparing to any of STs in existing CCs in the database [20]. ODC is an extension of the CC 139 clustering method, but it was performed only at MGT9 level [20]. For example, any two MGT9 STs 140 with no more than 2, 5, or 10 allele differences were assigned the same ODC2, ODC5 or ODC10 141 type [20].

\section{Geographic distribution and temporal variation analysis}

143 We first determined which MGT level would be the most useful in describing the global 144 epidemiology of $S$. Enteritidis by summarizing STs that contained at least 10 isolates for their 145 distribution across continents. STs where more than $70 \%$ of isolates were from a single continent 146 were identified at each level of MGT (Fig. 2a). MGT4 STs matching this criterion had the highest 147 percentage of all the levels at $76.3 \%$ of the 26,670 isolates, thus MGT4 was chosen to describe the 148 continental distribution of $S$. Enteritidis. The USA states variation map and the UK monthly variation 149 column chart were produced using Tableau v2019.2 [31].

\section{Antibiotic resistance gene and plasmid specific gene identification}

151 Abricate v1.0.1 (https://github.com/tseemann/abricate) was used for identification of antibiotic 152 resistance genes with the ResFinder v3.0 database [32], and plasmid specific genes with the 
153 PlasmidFinder v2.1 database [33]. The cut-off of the presence of a gene was set as both identity and 154 coverage $>=90 \%$ [32]. At each MGT level, STs ( $>=10$ isolates each) with more than $80 \%$ of isolates 155 harbouring antibiotic resistance genes, were collected from MGT2 to MGT9 and were defined as 156 high-antibiotic-resistant STs. The STs at each level of MDR were non-redundant meaning that no 157 isolate was counted more than once.

\section{Phylogenetic analysis}

A phylogenetic tree was constructed using Parsnp v1.2, which called core-genome SNPs [34, 35]. Potential recombinant SNPs were removed using both Gubbins v2.0.0 and Recdetect v6.0 [36, 37]. Beast v1.10.4 was then used to estimate the mutation rate based on mutational SNPs [38]. A total of 24 combinations of clock and population size models were evaluated with the MCMC chain of 100 million states. Tracer v1.7.1 was used to identify the optimal model and to estimate population expansion over time [39]. The detailed methods for phylogenetic analysis were described in

\section{Supplementary material.}

\section{Virulence genes and Salmonella pathogenicity islands (SPIs) distribution in the $S$. Enteritidis}

\section{population}

We compared the presence of virulence determinants in the main lineages of $S$. Enteritidis. Virulence genes from the Virulence Factor Database (VFDB) were identified in all the $S$. Enteritidis genomes using Abricate v1.0.1 (https://github.com/tseemann/abricate), with identity threshold of $70 \%$ and coverage of 50\% [40]. Using the same blast threshold, a total of 23 reported SPIs from SPI-1 to SPI23 were also identified in all the $S$. Enteritidis genomes [41].

\section{Evaluation of the effect of repeat sequencing on the dataset for epidemiological analysis}

To evaluate any bias that may be caused by resequencing of the same isolate, we identified all isolates of the same ST based on MGT9 and same metadata based on collection country, collection 
176 year and month, and source type. Such isolates were conservatively treated as repeat sequencing of

177 the same isolate and such "duplicates" were removed from the dataset. We re-analysed the reduced

178 dataset and compared against the original dataset by calculating Kendall's tau [42]. The detailed

179 results are shown in Supplementary material and Data Set S2, Tab 1 to Tab 6.

180

181

182

\section{Results}

\section{Establishing the MGT system for $S$. Enteritidis}

The core genome of $S$. Enteritidis was defined at $>=96 \%$ identity and presence rate of $>=99 \%$ of the 1801 sampled isolates (Fig. S1). The core genome of $S$. Enteritidis included 3932 genes, with 977 not found in the Salmonella enterica core as well as 1054 S. Enteritidis core intergenic regions (Fig. 1a) (Data Set S1, Tab 2). We also searched for the presence of small RNA (sRNA) in the intergenic regions with $37(32.7 \%, 37 / 113)$ sRNAs observed in $36(3.4 \%, 36 / 1054)$ intergenic regions.

\section{The first eight schemes of $S$. Enteritidis MGT used the same 2955 core genes of Salmonella} enterica as the MGT of $S$. Typhimurium, and the rationale of locus selection has been fully described by Payne et al [20]. MGT1 corresponded to the classic seven gene MLST of Salmonella [12]. A total of 4986 loci were incorporated in the MGT9 scheme, including the $3932 S$. Enteritidis core genes and 1054 core intergenic regions defined (Fig. 1a).

A total of 26,670 $S$. Enteritidis genomes with publicly available raw reads were analysed using the MGT scheme. These publicly available genomes were collected from 26 source types with $49.9 \%$ of isolates collected from humans and $8.9 \%$ from avian sources; they were collected from 86 countries with the majority of the isolates from the United States (47.3\%) and United Kingdom (35.9\%); and they were collected between the years 1917 and 2018 with 2014 (11.5\%), 2015 (14.6\%), 2016 (14.0\%), 2017 (12.8\%) and 2018 (3.7\%) having more than 500 isolates each (Fig. S2a). At each MGT level, each isolate was assigned an ST and a clonal complex (CC). A CC in this study was 
199 defined as a group of STs with one allele difference [12, 43]. The number of STs and CCs at each

200 MGT level is shown in Fig. 1b. As the resolution of typing increased from MGT1 to MGT9, an

201 increasing number of STs and CCs were assigned. At MGT2, the 26,670 isolates were subtyped into

202252 STs and 23 CCs. By contrast, MGT9 divided the isolates into 20,153 different STs and 14,441

203 CCs. The MGT scheme of $S$. Enteritidis is available through a public online database

204 (https://mgtdb.unsw.edu.au/enteritidis/).

\section{The international or global epidemiology of $S$. Enteritidis by MGT}

Of the 26,670 isolates typed by MGT, 25,207 have country metadata. By the 7-gene MLST (or MGT1) scheme, ST11 was the dominant type representing $94.8 \%$ of the isolates, followed by ST183 representing $1.6 \%$. ST183 is an endemic ST prevalent in Europe and was divided into two main phage types, PT11 and PT66 [44]. Using MGT, ST183 (or MGT1-ST183) can be divided into seven STs at MGT2 level. Interestingly, 97\% (137/141) of the PT11 isolates belonged to MGT2-ST3, and $100 \%(24 / 24)$ of the PT66 belonged to MGT2-ST82, highlighting the potential for backward

212 compatibility of MGT with traditional typing data.

For the predominant ST11 isolates (or MGT1-ST11), we found that the optimal MGT level to

214 describe their global epidemiology was MGT4, based on the distribution of each ST in different 215 continents (Fig. 2a). At the MGT4 level, the 26,670 isolates were subtyped into 2,236 STs and 423

216 CCs. Among the 2,236 MGT4-STs, 163 STs were predominantly found in only one continent 217 (defined as $\geq 70 \%$ from one continent). These 163 STs contained 20,341 of the 26,670 genomes $218(76.3 \%)$.

219 The distribution of STs varied between continents. The following MGT4-CC1 STs, ST171, ST163, 220 ST370, ST1009, and MGT4-CC13 STs, ST99, ST136, ST135, ST396, ST198, ST160, ST416 were 221 the most prevalent STs in North America (Fig. 2b). While in Europe, the following MGT4-CC1 STs, 222 ST15, ST208, ST357, ST237 and MGT4-CC13 STs, ST25, ST13, ST100, ST31, ST29 were 
223 common. However, the North America and Europe $S$. Enteritidis sequences analysed were mostly

224 obtained from the USA and UK, which represented $94.6 \%$ and $92.5 \%$ of isolates from these two 225 continents (Fig. S2a). In Africa, MGT4-ST11 (also described by MGT3-ST10) was more prevalent 226 in West Africa and MGT4-ST16 (also described by MGT3-ST15) in Central/Eastern Africa (Fig. 227 S3). Finally, MGT4-ST15 was a global ST which was observed in all continents (Fig. 2b).

These dominant STs can be further grouped into CCs which offered a more inclusive picture. 229 Among the 423 MGT4-CCs at MGT4, 10 represented $94.1 \%$ of all the isolates. The top two CCs, 230 MGT4-CC1 and MGT4-CC13 accounted for $88.0 \%$ of the isolates (Fig. 2b). MGT4-CC1 was 231 prevalent in all six continents, while MGT4-CC13 was more common in North America and Europe 232 (Fig. 2b, Fig. S4).

\section{The national or local epidemiology of $S$. Enteritidis by MGT}

As the majority of the $S$. Enteritidis sequences analysed in this study were from the USA and UK, we compared the distribution of the STs between these two countries. We used MGT4 and MGT5 levels to describe the data. A total of 39 MGT4 STs with more than 50 isolates represented $75.1 \%$ of the USA and UK isolates and each country had its own specific types (Fig. 3a). MGT4-ST99, ST136, ST135, ST171, ST163, ST370, ST396 and ST150 were the main STs in the USA, whereas MGT4ST15, ST25, ST13, ST100, ST31, ST326, ST24, ST208, ST29 were the main STs in the UK. MGT4ST15 and ST25 were further subtyped into 34 MGT5-STs (each with more than 20 isolates), of which the majority were mainly observed in the UK, except for MGT5-ST412 and ST387 in the USA (Fig. 3b).

To examine the relationship between MGT-STs, isolation source and location, we examined MGT4-STs of 4383 genome sequences from the USA which contained source and state metadata. Of the 4383 genomes, $46.7 \%$ were from avian source while $43.1 \%$ were from humans. Six MGT4 STs (with more than 50 isolates each) were isolated from both human and avian sources including 
247 MGT4-ST99, ST135, ST136, ST160, ST198 and ST25, while three STs including MGT4-ST15,

248 ST163 and ST171 were mainly from human sources (Fig. 4). All of the human-only STs belonged to

249 MGT4-CC1 whereas the mixed source STs were mostly of MGT4-CC13 origin. STs belonging to

250 MGT4-CC13 were significantly more prevalent in avian sources than STs of MGT4-CC1 $(\mathrm{P}<0.001$,

251 OR = 45.9). For MGT4-CC13, S. Enteritidis isolate metadata from 48 states of the USA were

252 available and ST frequencies were similar across the country (Fig. 4). In almost all states, MGT4-

253 ST99 (within MGT4-CC13) was the dominant type, followed by MGT4-ST135 and MGT4-ST136.

254 While for MGT4-CC1 STs, ST15, ST163 and ST171 are predominant in only two states.

255 Most UK isolates contained collection year and month metadata. There were 8,818 human $S$.

256 Enteritidis isolates collected from March 2014 to July 2018 in the UK. We chose MGT5-STs to

257 describe the monthly variation of $S$. Enteritidis in the UK. By MGT5, variation in the prevalence of

258 MGT5-STs in different years and months was observed (Fig. 5). There were 13 MGT5-STs with

259 more than 100 isolates, representing $46.3 \%$ of the 8,818 isolates. The top five STs over the entire

260 period were MGT5-ST1, ST29, ST79, ST15 and MGT5-ST33, representing 30\% of the total isolates.

261 These STs showed differing temporal patterns. MGT5-ST1, ST29, ST79 and ST15 were consistently

262 observed in each month across these four years while MGT5-ST15 included isolates previously

263 reported as part of an outbreak [45]. MGT5-ST156 first appeared in April of 2012 in the UK,

264 increased in frequency in June and July of 2014, and became rare after 2014. MGT5-ST423 was a

265 dominant type from March to September of 2015, then became rare and was dominant again from

266 the September of 2016 to January of 2017.

\section{Detection of potential large outbreak clusters of $S$. Enteritidis using MGT}

268 MGT9 offered highest resolution for tracking strain spread and outbreak detection. By MGT9, there

269 were 124 MGT9-STs including isolates from two or more countries each, indicating international 
271 isolates using MGT9 allele differences with a range of cut-offs (0, 1, 2, 5 and 10 allelic differences)

272 that were named as outbreak detection clusters (ODC0, 1, 2, 5 and 10). These clusters can be used to 273 analyse frequencies of closely related isolates at different cut-off levels for population studies and 274 may also be used as dynamic thresholds to detect potential outbreaks [46].

275 In this study, we used ODC2 clusters to detect potential outbreak clusters and to assess whether 276 some STs were more likely to cause large outbreak clusters, based on the number of ODC2 clusters 277 and the total number of isolates in these clusters in different STs. Since the global data may be biased 278 towards outbreak isolates that were preferentially sequenced, we used UK data from 2014 to 2018 279 which included all human isolates referred to public health authorities [47]. There were 17 ODC2 clusters of more than 50 isolates representing 1855 isolates in total. The majority of these ODC2 clusters belonged to 12 different MGT4-STs and therefore we used MGT4 level to perform comparison (Data Set S1, Tab 4). MGT4-ST15 (1804 isolates) was the dominant ST in UK and

283 contained only one ODC2 cluster of 62 isolates while MGT4-ST25 (1532 isolates), which was the 284 second dominant ST in UK, contained five large ODC clusters containing 58 to 287 isolates (602 in 285 total). MGT4 ST15 is significantly less likely to contain large ODC2 clusters than the other STs (OR $286=0.1, \mathrm{P}$ value $<0.001)$, while MGT4-ST25 is significantly more likely to contain larger ODC2 287 clusters than other STs $(\mathrm{OR}=3.1$, P value < 0.001$)$. It is noteworthy that by clonal complexes, all of 288 the six top STs belonged to MGT4-CC13 and were positively associated with large scale outbreaks, 289 including three previously reported large scale outbreaks in Europe [19, 45, 48, 49]. Indeed, MGT4290 CC13 was significantly more likely to cause larger scale outbreaks than MGT4-CC1 $(\mathrm{OR}=6.2, \mathrm{P}<$ 291 0.001). These associations of STs and CCs with large outbreak clusters were also significant when 292 ODC5 clusters was used. Notably the cluster sizes were larger but the trend remains the same (Data 293 Set S1, Tab 4 and Tab 5). 
295 As nearly all of the isolates $(99.98 \%, 26,665 / 26,670)$ harboured the aac $\left(6^{\prime}\right)$-Iaa_l gene for 296 aminoglycoside resistance, this gene was excluded from the antibiotic resistance analysis. A total of 2972505 isolates (9.4\% of the total 26,670 isolates) were found to harbor antibiotic resistant genes 298 excluding $a a c\left(6^{\prime}\right)-I a a_{-}$. The most frequent predicted class was tetracyclines $(5.1 \%, 1350 / 26,670)$, 299 followed by beta-lactams $(4.5 \%, 1213 / 26,670)$ and aminoglycosides $(2.8 \%, 741 / 2270)$. Among the 3002505 isolates with AR genes, 40.4\% (1011/2505) were predicted to be MDR, harbouring genes 301 conferring resistance to three or more different antibiotic classes. And 59.6\% (1494/2505) of the 302 isolates harboured genes conferring resistance to one to two different antibiotic classes. Although the selection of isolates for genome sequencing in some continents may be biased, African isolates were found to have the highest proportion of AR/MDR isolates, followed by Asia and Oceania (Fig. S5a). AR associated STs (defined as $>=80 \%$ of the isolates are predicted to be MDR or AR). Eleven STs from varied levels were identified as MDR STs, representing 49\% (659/1011) of the MDR isolates. These STs were mutually exclusive at different MGT levels. The top two STs, MGT3-ST15 and ST10, were the two invasive types that were prevalent in Africa. For MGT3-ST15, 99.2\% of the isolates harboured resistance genes corresponding to as many as six different drug classes (Table 1). For MGT3-ST10, $86.5 \%$ of the isolates were MDR, and the antibiotic resistant patterns were similar to those of MGT3-ST15. MGT3-ST10 and ST15 represented 96.3\% and 90.2\% of the two Africa endemic lineages (MGT3-CC10 and CC15, respectively) as mentioned below. Among the other nine

314 MDR STs, eight belonged to MGT4-CC1, and 93.5\% of the isolates (243/260) harboured genes 315 conferring resistance to as many as eight classes of antibiotic drugs. Only one MDR ST (MGT6316 ST2698) belonged to MGT4-CC13 with 89.7\% (35/39) of the isolates harboured genes conferring 317 resistance to four drug classes. Based on available population sampling, MGT4-CC1 had 318 significantly more isolates with MDR genes (5.6\%, 474/8539) than MGT4-CC13 $(0.7 \%, 107 / 14,972)$ 319 (Fisher exact test, $\mathrm{P}$ value $<0.001, \mathrm{OR}=12.4$ ). 
A total of 707 isolates belonging to 47 different STs from MGT3 to MGT7, harboured genes conferring resistance to one or two different antibiotic classes including aminoglycosides, betalactams, tetracyclines and quinolones (Data Set S1, Tab 6). Among the 47 STs, 34 (72\%, 34/47) belonged to MGT4-CC1, representing 80\% (564/707) of isolates, and 12 (26\%, 12/47) STs belonged to MGT4-CC13 representing $18 \%(125 / 707)$ of the isolates. Again MGT4-CC1 had significantly more isolates with AR genes than MGT4-CC13 (Fisher exact test, P value < 0.001, OR = 8.4).

Plasmid specific genes were identified from the PlasmidFinder database [33] and IncQ1, IncN, IncI1, IncX1 plasmid types were common in $S$. Enteritidis isolates with AR genes (Fig. S5b). In particular, plasmid type IncQ1 was present in MGT3-ST15, ST10 and ST30, which harboured MDR genes up to six drug classes (Table 1). Plasmid type IncI1 was common in MGT3-ST10 and ST161 and plasmid type IncX1 was more common in the STs of MGT4-CC1.

\section{The population structure and evolution of major $S$. Enteritidis STs/CCs}

The majority of the STs from different MGT levels analysed belonged to the two MGT4 CCs, MGT4-CC1 and CC13. To describe the global phylogenetic structure of $S$. Enteritidis and explore the phylogenetic relationship of the two lineages, 1506 representative isolates were selected using representatives of MGT6-STs to encompass the diversity of the serovar. A previous study had suggested that $S$. Enteritidis has three clades, A, B and C. Clade A and C appeared to diverge earlier and were phylogenetically more distant to the global clade B than Salmonella enterica serovar Gallinarum [11] (Fig. 6a). S. Enteritidis clade B and $S$. Gallinarum were sister clades. The vast majority of the genomes analysed belonged to clade B.

Within clade B, we identified 10 main lineages, which were concordant with MGT-CCs from MGT1 to MGT4 (Fig. 6b). These lineages can be described at different MGT levels as shown in Fig. 6b. The lineages were consistent with the progressive division from lower to higher MGT levels grouped by CCs. MGT1-ST11 can be represented by MGT2-CC1 and MGT1-ST183 by MGT2-CC3. 
344 At MGT3, four lineages named MGT3-CC10, CC15, CC18 and CC107 defined separate lineages, while MGT3-CC1 included several lineages represented by MGT4-CCs. MGT3-CC10 and CC15 represented $100 \%$ of the two previously reported Africa lineages associated with invasive infection

347 [5]. MGT3-CC1 included five main MGT4-CCs, with two, MGT4-CC1 and MGT4-CC13, 348 representing $88.0 \%$ of all 26,670 S. Enteritidis isolates (Fig. 6b). MGT4-CC30 and CC129, both of 349 which were phylogenetically closer to MGT3-CC13, were two endemic lineages in Europe and 350 North America, respectively (Fig. 6b). The population structure of $S$. Enteritidis defined by MGT, were generally in accordance with cgMLST HierCC HC100 (Fig. S6). The association between MGT-STs/CCs with previously reported SNP analysis-based nomenclature (lineages/clades) and phage types were summarized (Dataset 1 , Tab 7) $[5,19,44,45,49]$. rate of the clade B $S$. Enteritidis which was estimated to be $1.9 \times 10^{-7}$ substitution/site/year (95\% CI of $1.6 \times 10^{-7}$ to $\left.2.3 \times 10^{-7}\right)$, corresponding to $0.8 \mathrm{SNPs}$ per genome per year for the core genome (95\% CI of 0.6 to $0.9 \mathrm{SNPs}$ ). The mutation rate of MGT4-CC13 lineage core genome was estimated to be $2.5 \times 10^{-7}$ substitution/site/year $\left(95 \% \mathrm{CI}\right.$ of $2.3 \times 10^{-7}$ to $\left.2.7 \times 10^{-7}\right)$ or $1.0 \mathrm{SNP}$ per genome per year

359 (95\% CI of 0.9 to $1.1 \mathrm{SNPs}$ ). The mutation rate of MGT4-CC1 lineage core genome was $1.7 \times 10^{-7}$ substitution/site/year $\left(95 \% \mathrm{CI}\right.$ of $1.6 \times 10^{-7}$ to $\left.2.0 \times 10^{-7}\right)$, or 0.7 SNP per genome per year (95\% CI of 0.6 to 0.8 SNPs). The mutation rate of MGT4-CC13 was significantly faster (1.5 times) than that of MGT4-CC1.

The most recent common ancestor (MRCA) of the nine lineages belonging to MGT2-CC1, was estimated to have existed in the 1460s (95\% CI 1323 to 1580) (Fig. S7). The two global epidemic 365 lineages MGT4-CC1 and CC13 are estimated to have diverged at around 1687 (95\% CI 1608 to 366 1760). In 1869 (95\% CI=1829-1900), MGT4-CC13 diverged into two sub-lineages of various 367 MGT4-STs, with one more prevalent in North America than in other continents (labelled with red arrow) and the other sub-lineage more prevalent in Europe (labelled with blue arrow). We further 
369 estimated the population expansion of the two global epidemic lineages MGT4-CC1 and CC13 (Fig. 7a, b). For MGT4-CC1, there were two large expansions around 1950 and 1970. For MGT4-CC13,

371 the population gradually increased until a more rapid expansion around 1970.

\section{Virulence genes and SPIs distribution in the STs/CCs represented phylogeny of $S$. Enteritidis}

373 We further compared the distribution of virulence genes and SPIs in the 13 STs/CCs that represent 374 the major phylogenetic lineages of $S$. Enteritidis. Based on the VFDB database, 162 genes were 375 present in >= 10 isolates of $S$. Enteritidis, $123(75.9 \%)$ genes of which were present in all of the 376 STs/CCs. Sixteen genes (9.9\%) were associated with one or more STs/CCs while the remainder were 377 sporadically distributed (Table 2). The $s p v$ locus including $s p v B, s p v C$ and $s p v R$, are reported to be associated with non-typhoidal bacteraemia [50]. SpvBCR genes were absent in MGT1-ST3304, ST180, ST1972 but present in all the other CCs. Pef fimbriae operon pefABCD genes were absent in MGT1-ST3304, ST180, ST1972, ST183 and MGT3-CC10. The ssek2 gene, which encodes a secretion effector of SPI-2, was reported to significantly contribute to the pathogenicity of Salmonella [51]. ssek2 was present in MGT3-CC107 and CC18, MGT4-CC129 and CC1, but was absent in MGT4-CC13 and other STs/CCs. On the reference genome P125109 (MGT4-CC1), ssek2 was observed in the prophage $\square$ SE20. Moreover, 34\% of the isolates in MGT3-CC107 (further represented by five STs from MGT4 to MGT7 levels) were found to harbour the Yersinia highpathogenicity island (HPI), representing $75.5 \%$ of the HPI positive isolates (Data Set S1, Tab 8). STs/CCs, and SPI-8, 15, 18, 20 and 21 were absent in all STs/CCs (Table 2). SPI-1 was intact in MGT1-ST1972 (clade C), while all the other STs/CCs had three SPI-1 genes (STM2901, STM2902 and STM2903) missing. SPI-5 was nearly intact in all STs/CCs with one to two genes missing in MGT1-ST183 and MGT4-CC101. SPI-11 was only intact in MGT4-CC101, with four to five genes missing in the other STs/CC. SPI-17 was intact in the majority of the STs/CCs except for MGT1- 
393 ST3304 and ST180 (clade A) with only one gene observed. SPI-19 was nearly intact in MGT1-

394 ST3304, ST180, ST1972 and ST183, but was truncated in all the other CCs in clade B. None of the

395 STs/CCs harboured the intact SPI-6, 7, 10, 22, and 23, only a few genes of which were observed.

396 MGT1-ST183, MGT3-CC15 and MGT4-CC13 were found to harbour 44 to 49 genes of SPI-7 (149

397 in total), the majority of which were located on the Fels2-like prophage.

\section{Discussion}

$S$. Enteritidis is one of the most common foodborne pathogens causing large scale national and international outbreaks and food recalls $[3,52]$. Understanding the population structure and genomic epidemiology of $S$. Enteritidis is essential for its effective control and prevention. In this study, we applied the genomic typing tool MGT to $S$. Enteritidis and developed a database for international applications. We used MGT to describe its local and global epidemiology, and its population structure using 26,670 publicly available genomes and associated metadata. In this work, STs and CCs were assigned to each of the nine MGT levels, with MGT1 refers to the legacy seven gene MLST [12]. Thus, attention should be paid to the prefix MGT levels for those STs and CCs to avoid confusion.

\section{The $S$. Enteritidis MGT scheme enables a scalable resolution genomic nomenclature}

The design of the $S$. Enteritidis MGT is based on the $S$. Typhimurium MGT scheme published previously [20]. The first eight levels (MGT1 to MGT8) of $S$. Enteritidis MGT scheme used the same loci as for $S$. Typhimurium. We defined the core genome of $S$. Enteritidis which had 3932 core genes and 1054 core intergenic regions, which was used as MGT9. MGT9 substantially increased the subtyping resolution for $S$. Enteritidis [13]. Our study suggests that the MGT levels one to eight could be applied to all Salmonella enterica serovars as a common scheme for the species, and only an additional serovar-specific MGT9 scheme needs to be designed for individual serovars that require the highest resolution for outbreak investigations. 
417 The online database of the $S$. Enteritidis MGT scheme offers an open platform for global

418 communication of genomic data and facilitates detection of international transmission and outbreaks

419 of $S$. Enteritidis. The STs, especially at the middle resolution MGT level, were associated with

420 different geographic regions, sources and MDR. The extension of MGT to $S$. Enteritidis was based

421 on our previous study on S. Typhimurium [20]. Importantly, the stable characteristics of STs, which

422 are based exact comparison, makes up for the main drawback of single-linkage clustering methods

423 that the cluster types may change, especially at higher resolutions. MGT STs enables long term

424 epidemiological communication between different laboratories. And the variable resolution of MGT

425 offers flexibility for temporal and spatial epidemiological analysis using an underlying stable

426 nomenclature of STs from the finest resolution level. Additionally, CCs at each MGT level were able

427 to cluster the STs with one allele difference and are concordant with phylogenetic lineages as shown

428 in this study.

MGT for $S$. Enteritidis uncovers geographic, source and temporal epidemiological trends of $S$.

430 Enteritidis within and between countries

431 A total of 26,670 isolates were successfully typed using the MGT scheme which allowed the 432 examination of the global (or international) and local (or national) epidemiology of $S$. Enteritidis.

433 The salient feature of the flexibility of the different levels of MGT has also been illustrated through

434 this analysis. The lower resolution levels of MGT were found to be able to effectively describe the 435 geographic variation in different continents, countries or regions. Of these levels we showed that 436 MGT4-STs best described the global epidemiology of $S$. Enteritidis at continental level with some 437 STs distributed globally while others more geographically restricted. MGT4-ST15 was a global 438 epidemic type which was prevalent in almost all continents. By contrast, MGT4-ST16 (also 439 described by MGT3-ST15) was prevalent in Central/Eastern Africa and MGT4-ST11 (also described by MGT3-ST10) was prevalent in West Africa, which agreed with a previous study [5]. 
441 In USA, MGT4-CC13 and MGT-CC1 showed remarkable difference in their epidemiology.

442 MGT4-CC13 STs including MGT4-ST99, ST136 and ST135 were the main cause of clinical

443 infections and were commonly isolated from poultry. The prevalent STs were generally similar in

444 different states. In particular, MGT4-ST99, ST136 and ST135 were the dominant types in almost all

445 states of the USA. The distribution and poultry association of these STs suggest that they may be

446 responsible for several multistate outbreaks caused by $S$. Enteritidis contaminated eggs [1, 53]. Since

447 poultry related products (i.e. eggs, chicken and turkey, especially eggs) are known to be the main

448 source of $S$. Enteritidis infections or outbreaks in the USA [4], this isn't surprising, but it 449 demonstrates the utility of the MGT.

450 On the other hand, MGT4-CC1 including MGT4-ST171, ST15 and ST163, were less prevalent in 451 the USA. Nevertheless, these STs were isolated from human infections but were very rare in poultry, 452 although sampling bias may affect this conclusion as the poultry isolates in the dataset was not from 453 systematic sampling of poultry sources. On the other hand, beef, sprouts, pork, nuts and seeds can 454 also be contaminated by $S$. Enteritidis [4]. These non-poultry related foods may be the source of the 455 MGT4-CC1 caused infections. Overall MGT4-CC13 was found to be significantly associated with 456 poultry source, which is concordant with a previous study [10]. Further studies are required to 457 explain the association of MGT4-CC13 but not MGT4-CC1 with poultry products. The lack of 458 potential source isolates within MGT4-CC1 STs highlights the need for comprehensive sequencing 459 and epidemiological efforts across the food production chain. Machine learning approaches, which 460 have been applied to the root source identification for $S$. Typhimurium outbreaks, could also 461 facilitate the source tracing of $S$. Enteritidis [17].

462 Temporal variation of UK $S$. Enteritidis was depicted clearly by MGT. From 2014 onwards, all 463 clinical $S$. Enteritidis were routinely sequenced in the UK [47]. Some MGT5-STs were found to 464 occur for a few months and then disappear and may be indicative of outbreaks. For example, MGT5- 
465 ST156, which increased substantially during June and July in 2014 but became rare after 2015 and 466 describes a reported large scale outbreak [19]. Other STs persisted for long periods of time. For 467 example, MGT5-ST1 and ST29, appeared to be endemic isolates which may be associated with local 468 reservoirs. Therefore, the variation of MGT-STs across different seasons offered additional 469 epidemiological signals for suspected outbreaks and endemic infections of $S$. Enteritidis. The 470 stability of STs avoids the potential cluster merging issues of single linkage clustering based methods 471 and ensures continuity for long-term surveillance [20].

\section{MGT for $S$. Enteritidis facilitates outbreak detection, source tracing and evaluation of} outbreak propensity

Whole genome sequencing offered the highest resolution for outbreak detection and source tracing. However, resolution of cgMLST heavily depends on the diversity of the species. Our previous study showed that for $S$. Typhimurium, serovar core genome offered higher resolution than species level core genome for outbreak investigation [20, 54]. We designed MGT9 for S. Enteritidis using 4986 loci, which is around 2000 more loci than Salmonella enterica core genes [13, 20], thus increasing the resolution of subtyping.

To facilitate outbreak detection MGT9 STs were further grouped to ODCs. There is no agreed upon single cut-off for outbreak detection and dynamic thresholds have been suggested [46]. Here, we used ODC2, which has a two-allele difference cut-off, to identify potential outbreak clusters. Although the data do not allow us to confirm whether any of these ODC2 clusters were actual outbreaks, a number of known outbreaks from other studies fell into ODC2 clusters [46, 48, 55]. A two-allele cut-off was selected because it is at the lower end of cut-offs in reported Enteritidis outbreaks $[48,55]$, which should limit the number of false positive outbreak calls. However, due to the variability in diversity of isolates from different outbreaks, a dynamic threshold for cut-offs would be more sensitive and specific to detect outbreaks [46]. Further work for $S$. Enteritidis is 
489 required to address this issue fully. Using the UK clinical isolates from 2014 to 2018, we found that 490 the European and North American prevalent lineage MGT4-CC13 was significantly correlated with 491 larger ODC2 clusters (>= 50 isolates each) than the global epidemic lineage of MGT4-CC1. Thus, 492 MGT4-CC13 is more likely to cause large scale outbreaks than MGT4-CC1. In the past few years, 493 several large scale outbreaks in Europe were due to the contaminated eggs $[48,49,52]$; these 494 isolates all belonged to MGT4-CC13. In the USA, MGT4-CC13 was the dominant lineage in both 495 human infections and poultry product contamination, while MGT4-CC1 was relatively rare in 496 poultry. Industrialised and consolidated poultry/eggs production and marketing could have facilitated 497 the spread of MGT4-CC13 causing large scale outbreaks. Further studies are required to definitively 498 identify the biological and environmental mechanisms facilitating these large MGT4-CC13 499 outbreaks.

502 clustering for outbreak detection. All three methods use the same single linkage clustering method

503 for outbreak detection. MGT offers further advantage that potential outbreak clusters can be 504 precisely defined by a stable MGT-ST identifier rather than a cluster number [20].

\section{MGT for $S$. Enteritidis improves precision of detection and tracking of MDR clones globally}

506

507

508

509

510 511
The rise of AMR in Salmonella is a serious public health concern [5]. The global spread of AMR can be mediated by lateral transfer of resistance genes as well as clonal spread of resistant strains. This study systematically evaluated the presence of antibiotic resistant genes in the 26,670 genomes of $S$. Enteritidis, 9.4\% of which harboured antibiotic resistant genes. Among the two global epidemic lineages, MGT4-CC1 was found to contain significantly more antibiotic resistant isolates than MGT4-CC13. 
512 We further identified STs that were associated with MDR. Eleven STs (>= 10 isolates each, 659

513 isolates in total) of different MGT levels were associated with MDR. These STs from different MGT

514 levels were mutually exclusive, emphasising the flexibility of MGT for precise identification of

515 MDR clones. MGT3-ST15 and MGT3-ST10 were representative of the previously reported Africa

516 invasive infection related lineages [5], harboured resistant genes of up to six different antibiotic

517 classes. The other STs, which were mainly from Europe and North America, harbour AR genes to up

518 to eight different classes of drugs. MGT3-ST30 and MGT4-ST718, which belonged to MGT4-CC1

519 and were observed in Europe, had been reported to be MDR phenotypically [56]. Significantly,

520 isolates from these two STs were mainly isolated from blood samples [56]. These MDR STs

521 correlated with blood stream infections should be monitored closely as they can potentially be more

522 invasive [5].

523 Because plasmids are known to play a key role in the acquisition of drug resistance genes [57, 58],

524 we identified Inc plasmid groups that are likely present in the $S$. Enteritidis population. Several of 525 these putative plasmids were MDR associated. MDR of African isolates in MGT3-ST10 and ST15

526 has been reported to be mediated by plasmids [5]. The West Africa lineage MGT3-ST10 isolates

527 were reported to have IncI1 plasmids [59]. In this study, both IncI1 and IncQ1 plasmid were present

528 in the Africa MGT3-ST10 and ST15 isolates as well as STs belonged to global epidemic lineage

529 MGT4-CC1. IncQ1 plasmids, which are highly mobile and widely transferred among different 530 genera of bacteria [60], are correlated with MDR of Salmonella [61, 62]. IncI1 plasmids are 531 responsible for the cephalosporin resistance of Salmonella and Escherichia in several countries [63532 65]. Moreover, IncN and IncX1 plasmids were common in the MDR associated STs of MGT4-CC1 533 and CC13. The use of MGT could enhance the surveillance of drug resistance plasmids.

534 Defining the population structure of $S$. Enteritidis with increasing resolution and precision at 
536 In this study, we defined the global population structure of $S$. Enteritidis using MGT-CCs. Three

537 clades of $S$. Enteritidis were previously defined with clade A and C as outgroups to the predominant 538 clade B [11], a classification supported by this study. We further identified 10 lineages within clade

539 B, which can be represented by different CCs from MGT2 to MGT4 corresponding with time of 540 divergence. Lower resolution level MGT CCs described old lineages well while higher resolution 541 level MGT CCs described more recently derived lineages (Fig. S7). Thus, the epidemiological 542 characteristics of a lineage can be identified using STs and/or CCs at appropriate MGT level. MGT2-

543 CC3 represent the Europe endemic MGT1-ST183 lineage, within which MGT2-ST3 and MGT2-

544 ST82 were able to identify previously defined phage types PT11 and PT66 respectively [44]. MGT3-

545 CC10 included all of the reported West Africa lineage isolates, and MGT3-CC15 included all of the 546 reported Central/Eastern Africa lineage isolates as well as Kenyan invasive $S$. Enteritidis isolates [5, 547 8]. MGT3-ST10 and MGT3-ST15 (represented 96.3\% of MGT3-CC10 and 90.2\% of MGT3-CC15 548 respectively), were MDR with similar resistance patterns. Most importantly, the two major global 549 epidemic lineages are clearly described by MGT4-CC1 and MGT4-CC13. MGT4-CC1 corresponds 550 to the global epidemic clade in the study of Feasey et al., and MGT4-CC13 to the outlier clade of 551 that study [5]. MGT4-CC1 and CC13 correspond to lineage III and lineage V in Deng et al's study 552 [10]. Thus, by using STs and/or CCs of different MGT levels the population structure of $S$. 553 Enteritidis can be described clearly and consistently. expansion time and evolutionary dynamics. Bayesian evolutionary analysis revealed a core genome

556 mutation rate for $S$. Enteritidis of $1.9 \times 10^{-7}$ substitution/site/year which is similar to the genome 557 mutation rate of $2.2 \times 10^{-7}$ substitution/site/year estimated by Deng et al [10]. In contrast, the mutation rate of MGT4-CC13 was 1.5 times faster than MGT4-CC1. Population expansion of MGT4-CC1 occurred with two peaks in the 1950s and 1970s, while MGT4-CC13 population increased steadily until the 1970s. The expansion around the 1970s may have been driven by the development of the 
561 modern industrialised poultry industry, including poultry farms and/or processing plants, that $S$.

562 Enteritidis colonized. This is concordant with Deng et al's speculation that the expansion of $S$.

563 Enteritidis population was correlated with the poultry farm industry [10, 18].

564 Acquisition and degradation of virulence factors in the STs/CCs represent phylogeny of $S$.

565 Enteritidis

566 By screening the virulence genes and SPIs in different STs/CCs representing the three clades and 567 major lineages of $S$. Enteritidis, distributional differences were observed for some virulence genes 568 and SPIs. The main difference observed was between clade A/C and clade B, and MGT1-ST183 and 569 other lineages of clade B. SpvBCR, pefABCD, and $r c k$ genes were found to be located on the same 570 plasmid in the complete genomes of $S$. Enteritidis (data not shown). However, the Europe endemic 571 MGT1-ST183 and the West Africa endemic MGT3-CC10 were positive for the spvBCR genes, but 572 were negative for the pefABCD and $s p v B C R$ genes, suggesting that there may exist other 573 mechanisms of acquisition of $s p v B C R$ genes. Variation in the distribution of genes on SPIs was also 574 observed. Some virulence or SPIs genes were likely to have been lost in certain STs/CCs. 575 Degradation of virulence genes had been previously observed in $S$. Enteritidis and $S$. Typhimurium $576[5,66]$. In summary, both acquisition and degradation of virulence factors occurred in the evolution 577 of $S$. Enteritidis.

578 Moreover, the main differences in virulence factors between the two dominant lineages MGT4$579 \mathrm{CC} 1$ and $\mathrm{CC} 13$ were ssek 2 and the number of SPI-7 genes. ssek2, located on the prophage $\square$ SE20 580 was limited to MGT4-CC1 [5], and around 35 SPI-7 genes, located on the Fels2-like prophage were 581 limited to MGT4-CC13 [5]. $\square$ SE20 was found to contribute to mouse infections [67]. Fels2-like 582 prophage was also present in a bloodstream infection related lineage three of $S$. Typhimurium ST313 583 in Africa, which is estimated to have significantly higher invasiveness index than the other lineages 584 of ST313 [66]. However, the detailed pathogenic role of Fels2-like prophage in Salmonella 585 infections remained unclear. There are potentially undiscovered virulence factors contributing to the 
586 epidemiological variation (infection severity, outbreak propensity and geographic spreading) among 587 clades and lineages of $S$. Enteritidis.

\section{Limitations of this study and challenges for public database}

589 The epidemiological results generated in this study were based on the publicly submitted metadata 590 and the correctness of the results therefore depends on the accuracy of this data. In as many cases as 591 possible metadata were verified from published sources. However, the possibility exists that 592 incorrect metadata or repeated sequencing of the same isolates influence the results. We showed the

593 effect of the latter was minimal (Supplementary Material). However, good metadata is essential, 594 and an internationally agreed on minimal metadata would be useful for better epidemiological 595 surveillance of $S$. Enteritidis. The online MGT system offers unprecedented power for monitoring $S$. 596 Enteritidis across the globe. Good metadata further enhances that power. Moreover, all the genomes 597 of this work were Illumina short-read sequencing except for one reference complete genome. The 598 thresholds of identity and coverage for searching AR genes and plasmid replicon genes were $>=$ $59990 \%$. Incomplete genomes and these thresholds may result in some AR and plasmid replicon genes 600 being missed. Additionally, considering the mobile nature of plasmids and prophages, some STs may 601 lose AR or virulence genes in rare cases, timely updating of ST definitions with respect to AR and 602 virulence states will therefore be necessary.

\section{Conclusions}

604 In this study, we defined the core genome of $S$. Enteritidis and developed an MGT scheme with nine 605 levels. MGT9 offers the highest resolution and is suitable for outbreak detection whereas the lower 606 levels (MGT1 to MGT8) are suitable for progressively longer epidemiological timescales. At the 607 MGT4 level, globally prevalent and regionally restricted STs were identified, which facilitates the 608 identification of the potential geographic origin of $S$. Enteritidis isolates. Specific source associated 609 STs were identified, such as poultry associated MGT4-STs, which were common in human cases in 
610 the USA. At the MGT5 level, temporal variation of STs was observed in S. Enteritidis infections

611 from the UK, which reveal both long lived endemic STs and the rapid expansion of new STs. Using

612 MGT3 to MGT6, we identified MDR-associated STs to facilitate tracking of MDR spread.

613 Additionally, certain plasmid types were found to be highly associated with the same MDR STs,

614 suggesting plasmid-based resistance acquisition. Furthermore, we evaluated the phylogenetic

615 relationship of the STs/CCs by defining the population structure of $S$. Enteritidis. A total of 10 main

616 lineages were defined in the globally distributed clade B of $S$. Enteritidis, which were represented

617 with 10 STs/CCs. Of these, MGT4-CC1 and CC13 were the dominant lineages, with significant

618 differences in large outbreak frequency, antimicrobial resistance profiles and mutation rates. Some

619 virulence and SPIs genes were distributed differently among the different STs/CCs represented

620 clades/lineages of $S$. Enteritidis. The online open database for $S$. Enteritidis MGT offers a unified

621 platform for the international surveillance of $S$. Enteritidis. In summary, MGT for $S$. Enteritidis

622 provides a flexible, high resolution and stable genome typing tool for long term and short term

623 surveillance of $S$. Enteritidis infections.

\section{Authors' contributions}

625 L.L., M.P., and R.L. designed the study. L.L. and M.P. set up the database. S.K and M.P. set up the 626 website, M.P., R.L., D.H. L.C. S.O., Q.W., M.T., V.S. and S.K. provided critical analysis and

627 discussions, L.L. wrote the first draft and all authors contributed to the final manuscript.

\section{Funding information}

629 This work was funded by a project grant from the National Health and Medical Research Council of 630 Australia (grant number 1129713). Lijuan Luo was supported by a UNSW scholarship (University

631 International Postgraduate Award). The funders had no role in study design, data collection and 632 interpretation, or the decision to submit the work for publication. 


\section{Acknowledgements}

634 The authors thank Duncan Smith and Robin Heron from UNSW Research Technology Services for 635 high performance computing assistance.

\section{Conflicts of interest}

The authors declare that there are no conflicts of interest.

638

639

640

641

642

643

644

645

646

647

648

649

650

651

652

653

654

655

656

657

658

659

\section{References}

1. Dewey-Mattia D, Manikonda K, Hall AJ, Wise ME, Crowe SJ. Surveillance for Foodborne Disease Outbreaks - United States, 2009-2015. MMWR Surveill Summ 2018;67(10):1-11.

2. Salmonellosis - Annual Epidemiological Report for 2017 [database on the Internet]2020. Available from: https://www.ecdc.europa.eu/en/publications-data.

3. Pijnacker R, Dallman TJ, Tijsma ASL, Hawkins G, Larkin L et al. An international outbreak of Salmonella enterica serotype Enteritidis linked to eggs from Poland: a microbiological and epidemiological study. Lancet Infect Dis 2019;19(7):778-786.

4. Snyder TR, Boktor SW, M'Ikanatha N M. Salmonellosis Outbreaks by Food Vehicle, Serotype, Season, and Geographical Location, United States, 1998 to 2015. J Food Prot 2019;82(7):1191-1199.

5. Feasey NA, Hadfield J, Keddy KH, Dallman TJ, Jacobs J et al. Distinct Salmonella Enteritidis lineages associated with enterocolitis in high-income settings and invasive disease in low-income settings. Nat Genet, Article 2016;48(10):1211-1217.

6. Mohan A, Munusamy C, Tan YC, Muthuvelu S, Hashim R et al. Invasive Salmonella infections among children in Bintulu, Sarawak, Malaysian Borneo: a 6-year retrospective review. BMC Infect Dis 2019;19(1):330.

7. Thung TY, Mahyudin NA, Basri DF, Wan Mohamed Radzi CW, Nakaguchi Y et al. Prevalence and antibiotic resistance of Salmonella Enteritidis and Salmonella Typhimurium in raw chicken meat at retail markets in Malaysia. Poult Sci 2016;95(8):1888-1893. 
660

661

662

663

664

665

666

667

668

669

670

671

672

673

674

675

676

677

678

679

680

681

682

683

684

685

686

687

688

689

690

691

692

8. Akullian A, Montgomery JM, John-Stewart G, Miller SI, Hayden HS et al. Multi-drug resistant non-typhoidal Salmonella associated with invasive disease in western Kenya. PLoS Negl Trop Dis 2018;12(1):e0006156.

9. Parn T, Dahl V, Lienemann T, Perevoscikovs J, De Jong B. Multi-country outbreak of Salmonella enteritidis infection linked to the international ice hockey tournament. Epidemiol Infect 2017;145(11):2221-2230.

10. Deng X, Desai PT, den Bakker HC, Mikoleit M, Tolar B et al. Genomic epidemiology of Salmonella enterica serotype Enteritidis based on population structure of prevalent lineages. Emerg Infect Dis 2014;20(9):1481-1489.

11. Graham RMA, Hiley L, Rathnayake IU, Jennison AV. Comparative genomics identifies distinct lineages of $S$. Enteritidis from Queensland, Australia. PLoS One 2018;13(1):e0191042.

12. Achtman M, Wain J, Weill FX, Nair S, Zhou $\mathbf{Z}$ et al. Multilocus sequence typing as a replacement for serotyping in Salmonella enterica. PLoS Pathog 2012;8(6):e1002776.

13. Alikhan NF, Zhou Z, Sergeant MJ, Achtman M. A genomic overview of the population structure of Salmonella. PLoS Genet 2018;14(4):e1007261.

14. Zhou Z, Alikhan N-F, Mohamed K, Fan Y, Agama Study G et al. The EnteroBase user's guide, with case studies on Salmonella transmissions, Yersinia pestis phylogeny, and Escherichia core genomic diversity. Genome Res 2020;30(1):138-152.

15. Ashton P, Nair S, Peters T, Tewolde R, Day M et al. Revolutionising Public Health Reference Microbiology using Whole Genome Sequencing: Salmonella as an exemplar. bioRxiv 2015:033225.

16. Zhou Z, Charlesworth J, Achtman M. HierCC: A multi-level clustering scheme for population assignments based on core genome MLST. Bioinformatics 2021.

17. Zhang S, Li S, Gu W, den Bakker H, Boxrud D et al. Zoonotic Source Attribution of Salmonella enterica Serotype Typhimurium Using Genomic Surveillance Data, United States. Emerg Infect Dis 2019;25(1):82-91.

18. Deng X, Shariat N, Driebe EM, Roe CC, Tolar B et al. Comparative analysis of subtyping methods against a whole-genome-sequencing standard for Salmonella enterica serotype Enteritidis. J Clin Microbiol 2015;53(1):212-218.

19. Hormansdorfer S, Messelhausser U, Rampp A, Schonberger K, Dallman T et al. Reevaluation of a 2014 multi-country European outbreak of Salmonella Enteritidis phage type 14b using recent epidemiological and molecular data. Euro Surveill 2017;22(50):17-00196. 
693 20. Payne M, Kaur S, Wang Q, Hennessy D, Luo L et al. Multilevel genome typing:

694

695

696

697

698

699

700

701

702

703

704

705

706

707

708

709

710

711

712

713

714

715

716

717

718

719

720

721

722

723

724 genomics-guided scalable resolution typing of microbial pathogens. Euro Surveill 2020;25(20):1900519.

21. Gurevich A, Saveliev V, Vyahhi N, Tesler G. QUAST: quality assessment tool for genome assemblies. Bioinformatics 2013;29(8):1072-1075.

22. Robertson J, Yoshida C, Kruczkiewicz P, Nadon C, Nichani A et al. Comprehensive assessment of the quality of Salmonella whole genome sequence data available in public sequence databases using the Salmonella In silico Typing Resource (SISTR). Microb Genom 2018;4(2).

23. Bolger AM, Lohse M, Usadel B. Trimmomatic: a flexible trimmer for Illumina sequence data. Bioinformatics 2014;30(15):2114-2120.

24. Bankevich A, Nurk S, Antipov D, Gurevich AA, Dvorkin M et al. SPAdes: a new genome assembly algorithm and its applications to single-cell sequencing. $J$ Comput Biol 2012;19(5):455-477.

25. Souvorov A, Agarwala R, Lipman DJ. SKESA: strategic k-mer extension for scrupulous assemblies. Genome Biol 2018;19(1):153.

26. Wood DE, Salzberg SL. Kraken: ultrafast metagenomic sequence classification using exact alignments. Genome Biol 2014;15(3):R46.

27. Zhang S, Yin Y, Jones MB, Zhang Z, Deatherage Kaiser BL et al. Salmonella serotype determination utilizing high-throughput genome sequencing data. J Clin Microbiol, 10.1128/JCM.00323-15 2015;53(5):1685-1692.

28. Yoshida CE, Kruczkiewicz P, Laing CR, Lingohr EJ, Gannon VP et al. The Salmonella In silico Typing Resource (SISTR): An Open Web-Accessible Tool for Rapidly Typing and Subtyping Draft Salmonella Genome Assemblies. PLoS One 2016;11(1):e0147101.

29. Seemann T. Prokka: rapid prokaryotic genome annotation. Bioinformatics 2014;30(14):2068-2069.

30. Kroger C, Dillon SC, Cameron AD, Papenfort K, Sivasankaran SK et al. The transcriptional landscape and small RNAs of Salmonella enterica serovar Typhimurium. Proc Natl Acad Sci U S A 2012;109(20):E1277-1286.

31. Tableau (version. 9.1). J Med Libr Assoc 2016;104(2):182-183.

32. Zankari E, Hasman H, Cosentino S, Vestergaard M, Rasmussen S et al. Identification of acquired antimicrobial resistance genes. J Antimicrob Chemother 2012;67(11):2640-2644. 
725 33. Carattoli A, Zankari E, Garcia-Fernandez A, Voldby Larsen M, Lund O et al. In silico

726

727

728

729

730

731

732

733

734

735

736

737

738

739

740

741

742

743

744

745

746

747

748

749

750

751

752

753

754

755

756

757

758

detection and typing of plasmids using PlasmidFinder and plasmid multilocus sequence typing. Antimicrob Agents Chemother 2014;58(7):3895-3903.

34. Treangen TJ, Ondov BD, Koren S, Phillippy AM. The Harvest suite for rapid coregenome alignment and visualization of thousands of intraspecific microbial genomes. Genome Biol 2014;15(11):524.

35. Price MN, Dehal PS, Arkin AP. FastTree 2--approximately maximum-likelihood trees for large alignments. PloS one 2010;5(3):e9490-e9490.

36. Croucher NJ, Page AJ, Connor TR, Delaney AJ, Keane JA et al. Rapid phylogenetic analysis of large samples of recombinant bacterial whole genome sequences using Gubbins. Nucleic Acids Res 2015;43(3):e15.

37. Hu D, Liu B, Wang L, Reeves PR. Living Trees: High-Quality Reproducible and Reusable Construction of Bacterial Phylogenetic Trees. Molecular biology and evolution 2020;37(2):563-575.

38. Suchard MA, Lemey P, Baele G, Ayres DL, Drummond AJ et al. Bayesian phylogenetic and phylodynamic data integration using BEAST 1.10. Virus Evol 2018;4(1):vey016.

39. Rambaut A, Drummond AJ, Xie D, Baele G, Suchard MA. Posterior Summarization in Bayesian Phylogenetics Using Tracer 1.7. Syst Biol 2018;67(5):901-904.

40. Chen L, Yang J, Yu J, Yao Z, Sun L et al. VFDB: a reference database for bacterial virulence factors. Nucleic Acids Res 2005;33(Database issue):D325-328.

41. Mansour MN, Yaghi J, El Khoury A, Felten A, Mistou M-Y et al. Prediction of Salmonella serovars isolated from clinical and food matrices in Lebanon and genomic-based investigation focusing on Enteritidis serovar. International Journal of Food Microbiology 2020;333:108831.

42. Kendall MG. A new measure of rank correlation. Biometrika 1938;30(1/2):81-93.

43. Feil EJ. Small change: keeping pace with microevolution. Nat Rev Microbiol 2004;2(6):483495.

44. Lawson B, Franklinos LHV, Rodriguez-Ramos Fernandez J, Wend-Hansen C, Nair S et al. Salmonella Enteritidis ST183: emerging and endemic biotypes affecting western European hedgehogs (Erinaceus europaeus) and people in Great Britain. Sci Rep 2018;8(1):2449.

45. Kanagarajah S, Waldram A, Dolan G, Jenkins C, Ashton PM et al. Whole genome sequencing reveals an outbreak of Salmonella Enteritidis associated with reptile feeder mice in the United Kingdom, 2012-2015. Food Microbiol 2018;71:32-38. 
759 46. Payne M, Octavia S, Luu LDW, Sotomayor-Castillo C, Wang Q et al. Enhancing

760

761

762

763

764

765

766

767

768

769

770

771

772

773

774

775

776

777

778

779

780

781

782

783

784

785

786

787

788

789

790 genomics-based outbreak detection of endemic Salmonella enterica serovar Typhimurium using dynamic thresholds. Microb Genom 2019.

47. Chattaway MA, Dallman TJ, Larkin L, Nair S, McCormick J et al. The Transformation of Reference Microbiology Methods and Surveillance for Salmonella With the Use of Whole Genome Sequencing in England and Wales. Frontiers in public health, Original Research 2019;7(317):317.

48. Inns T, Lane C, Peters T, Dallman T, Chatt C et al. A multi-country Salmonella Enteritidis phage type $14 \mathrm{~b}$ outbreak associated with eggs from a German producer: 'near realtime' application of whole genome sequencing and food chain investigations, United Kingdom, May to September 2014. Euro Surveill 2015;20(16):21098.

49. Inns T, Ashton P, Herrera-Leon S, Lighthill J, Foulkes $\mathbf{S}$ et al. Prospective use of whole genome sequencing (WGS) detected a multi-country outbreak of Salmonella Enteritidis. Epidemiology \& Infection 2017;145(2):289-298.

50. Guiney DG, Fierer J. The Role of the spv Genes in Salmonella Pathogenesis. Front microbiol 2011;2:129-129.

51. Zhang X, He L, Zhang C, Yu C, Yang Y et al. The impact of sseK2 deletion on Salmonella enterica serovar Typhimurium virulence in vivo and in vitro. BMC Microbiol 2019;19(1):182.

52. Dallman T, Inns T, Jombart T, Ashton P, Loman N et al. Phylogenetic structure of European Salmonella Enteritidis outbreak correlates with national and international egg distribution network. Microb Genom 2016;2(8):e000070.

53. Nichols M, Stevenson L, Whitlock L, Pabilonia K, Robyn M et al. Preventing Human Salmonella Infections Resulting from Live Poultry Contact through Interventions at Retail Stores. J Agric Saf Health 2018;24(3):155-166.

54. Fu S, Octavia S, Tanaka MM, Sintchenko V, Lan R. Defining the Core Genome of Salmonella enterica Serovar Typhimurium for Genomic Surveillance and Epidemiological Typing. J Clin Microbiol 2015;53(8):2530-2538.

55. Taylor AJ, Lappi V, Wolfgang WJ, Lapierre P, Palumbo MJ et al. Characterization of Foodborne Outbreaks of Salmonella enterica Serovar Enteritidis with Whole-Genome Sequencing Single Nucleotide Polymorphism-Based Analysis for Surveillance and Outbreak Detection. J Clin Microbiol 2015;53(10):3334-3340. 
791 56. Vidovic S, An R, Rendahl A. Molecular and Physiological Characterization of

792 Fluoroquinolone-Highly Resistant Salmonella Enteritidis Strains. Front Microbiol $793 \quad 2019 ; 10: 729$.

794 57. Kaldhone PR, Carlton A, Aljahdali N, Khajanchi BK, Sanad YM et al. Evaluation of Incompatibility Group I1 (IncI1) Plasmid-Containing Salmonella enterica and Assessment of the Plasmids in Bacteriocin Production and Biofilm Development. Front Vet Sci 2019;6(298):298.

798

58. Zhou X, Li M, Xu L, Shi C, Shi X. Characterization of Antibiotic Resistance Genes, Plasmids, Biofilm Formation, and In Vitro Invasion Capacity of Salmonella Enteritidis Isolates from Children with Gastroenteritis. Microb Drug Resist 2019;25(8):1191-1198.

59. Aldrich C, Hartman H, Feasey N, Chattaway MA, Dekker D et al. Emergence of phylogenetically diverse and fluoroquinolone resistant Salmonella Enteritidis as a cause of invasive nontyphoidal Salmonella disease in Ghana. PLoS Negl Trop Dis 2019;13(6):e0007485.

60. Smalla K, Heuer H, Gotz A, Niemeyer D, Krogerrecklenfort E et al. Exogenous isolation of antibiotic resistance plasmids from piggery manure slurries reveals a high prevalence and diversity of IncQ-like plasmids. Appl Environ Microbiol 2000;66(11):4854-4862.

61. Castellanos LR, van der Graaf-van Bloois L, Donado-Godoy P, Leon M, Clavijo V et al. Genomic Characterization of Extended-Spectrum Cephalosporin-Resistant Salmonella enterica in the Colombian Poultry Chain. Front Microbiol 2018;9:2431.

62. Mastrorilli E, Pietrucci D, Barco L, Ammendola S, Petrin S et al. A Comparative Genomic Analysis Provides Novel Insights Into the Ecological Success of the Monophasic Salmonella Serovar 4,[5],12:i. Front Microbiol 2018;9:715.

63. Wong MH, Kan B, Chan EW, Yan M, Chen S. IncI1 Plasmids Carrying Various blaCTXM Genes Contribute to Ceftriaxone Resistance in Salmonella enterica Serovar Enteritidis in China. Antimicrob Agents Chemother 2016;60(2):982-989.

64. Garcia-Fernandez A, Chiaretto G, Bertini A, Villa L, Fortini D et al. Multilocus sequence typing of IncI1 plasmids carrying extended-spectrum beta-lactamases in Escherichia coli and Salmonella of human and animal origin. J Antimicrob Chemother 2008;61(6):1229-1233.

65. Kameyama M, Chuma T, Yokoi T, Yabata J, Tominaga $\mathbf{K}$ et al. Emergence of Salmonella enterica serovar infantis harboring IncI1 plasmid with bla(CTX-M-14) in a broiler farm in Japan. $J$ Vet Med Sci 2012;74(9):1213-1216. 
824 66. Pulford CV, Perez-Sepulveda BM, Canals R, Bevington JA, Bengtsson RJ et al.

825 Stepwise evolution of Salmonella Typhimurium ST313 causing bloodstream infection in 826 Africa. Nat Microbiol 2021;6(3):327-338.

827 67. Silva CA, Blondel CJ, Quezada CP, Porwollik S, Andrews-Polymenis HL et al. Infection

828 of mice by Salmonella enterica serovar Enteritidis involves additional genes that are absent 829 in the genome of serovar Typhimurium. Infect Immun 2012;80(2):839-849.

830

831

832

833

834

835

836

837

838

839 
Table 1. Antibimicrobial drug class and plasmid class of the MDR associated STs from different MGT levels.

\begin{tabular}{|c|c|c|c|c|c|c|c|c|c|c|c|c|c|c|c|c|c|c|c|c|}
\hline \multirow[b]{2}{*}{ MGT-ST $^{a}$} & \multirow[b]{2}{*}{$\begin{array}{l}\text { MGT4- } \\
\text { CCs }\end{array}$} & \multirow[b]{2}{*}{$\begin{array}{l}\text { Total } \\
\text { No. of } \\
\text { isolates }\end{array}$} & \multirow[b]{2}{*}{$\begin{array}{l}\text { No. of } \\
\text { isolates } \\
\text { with } \\
\text { MDR (\%) }\end{array}$} & \multicolumn{7}{|c|}{$\%$ of the MDR isolates ${ }^{d}$} & \multicolumn{9}{|c|}{$\%$ of isolates with plasmid ${ }^{d}$} & \multirow[b]{2}{*}{ Continent $^{e}$} \\
\hline & & & & 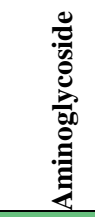 & 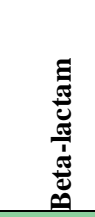 & 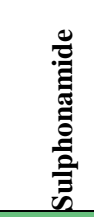 & 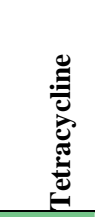 & : & 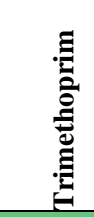 & 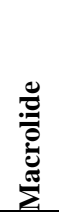 & 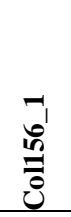 & 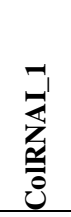 & $\begin{array}{l}u^{\prime} \\
\frac{1}{3}\end{array}$ & 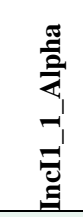 & 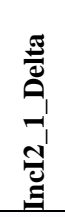 & $\vec{z}_{\mathfrak{\Xi}}$ & 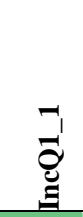 & $\begin{array}{l}I_{1} \\
\vec{x} \\
\dot{\Xi}\end{array}$ & 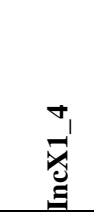 & \\
\hline MGT3-ST15 & $\mathrm{CC} 16^{c}$ & 261 & $259(99 \%)$ & $98 \%$ & $74 \%$ & $97 \%$ & $89 \%$ & $95 \%$ & $96 \%$ & & & $1 \%$ & & $14 \%$ & $1 \%$ & & $95 \%$ & & & Africa/EU/NA/OC \\
\hline MGT3-ST30 & $\mathrm{CC} 1$ & 10 & $10(100 \%)$ & $100 \%$ & & $100 \%$ & $100 \%$ & $100 \%$ & $100 \%$ & & & & & & & & $80 \%$ & & & EU/NA \\
\hline MGT3-ST161 ${ }^{b}$ & $\mathrm{CC} 1$ & 79 & $29(37 \%)$ & $30 \%$ & $84 \%$ & $35 \%$ & $84 \%$ & $32 \%$ & $3 \%$ & $3 \%$ & $1 \%$ & & $100 \%$ & $86 \%$ & & & & $1 \%$ & & EU \\
\hline MGT3-ST9 & $\mathrm{CC} 1$ & 70 & $66(94 \%)$ & $93 \%$ & $80 \%$ & $91 \%$ & $80 \%$ & $1 \%$ & $1 \%$ & $1 \%$ & $1 \%$ & & & $3 \%$ & & & & & $99 \%$ & EU/NA/OC \\
\hline MGT3-ST301 & $\mathrm{CC} 1$ & 11 & $11(100 \%)$ & $100 \%$ & $100 \%$ & & & & $100 \%$ & & & & & & & & & $100 \%$ & & NA/EU \\
\hline MGT4-ST354 & $\mathrm{CC} 1$ & 39 & $39(100 \%)$ & $100 \%$ & $100 \%$ & $100 \%$ & $62 \%$ & & & & $3 \%$ & & & $5 \%$ & & & & & $100 \%$ & EU/NA/OC \\
\hline MGT4-ST54 & $\mathrm{CC} 1$ & 29 & $28(97 \%)$ & $90 \%$ & $41 \%$ & $90 \%$ & $86 \%$ & & & & & & & & & & & & $97 \%$ & EU/NA/OC \\
\hline MGT5-ST86 & $\mathrm{CC} 1$ & 12 & $12(100 \%)$ & $100 \%$ & $100 \%$ & $100 \%$ & & & & & & & & $8 \%$ & & & & & $100 \%$ & NA/EU/OC \\
\hline MGT6-ST2698 & $\mathrm{CC} 13$ & 39 & $35(90 \%)$ & $90 \%$ & $90 \%$ & $90 \%$ & $90 \%$ & & & & & & & & & $90 \%$ & & $100 \%$ & & NA \\
\hline Total & & 701 & $621(89 \%)$ & & & & & & & & & & & & & & & & & \\
\hline
\end{tabular}

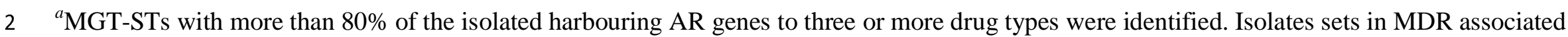

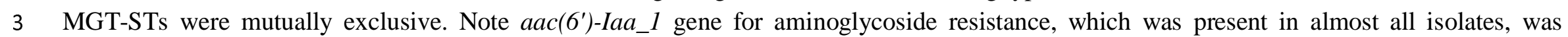
4 excluded for the AR analysis.

$5{ }^{b}$ MGT3-CC161 include 29 isolates with MDR genes (to five drug types) and 38 isolates with AR genes to two different drug types.

$6{ }^{c}$ MGT4-CC16 and CC11 belonged to MGT3-CC15 and CC10, which were representative of the two African lineages.

$7{ }^{d}$ The gradient green to gray colours reflect the proportion of isolates from $100 \%$ to $1 \%$.

$8{ }^{e}$ Continents in which the MDR related STs were observed: EU refers to Europe, NA to North America and OC to Oceania. 
Table 2. Virulence and SPI genes that distributed differently in the STs/CCs represented phylogeny of $S$. Enteritidis.

\begin{tabular}{|c|c|c|c|c|c|c|c|c|c|c|c|c|c|c|c|c|c|c|c|c|c|c|c|c|}
\hline \multirow[b]{3}{*}{ MGT1-ST3304 } & \multirow[b]{2}{*}{ Clade } & \multirow[b]{2}{*}{$\begin{array}{l}\text { No. of } \\
\text { isolates }\end{array}$} & \multicolumn{12}{|c|}{$\begin{array}{l}\text { The virulence gene present in }>80 \% \text { of the isolates in each } \\
\text { ST/CC }\end{array}$} & \multicolumn{10}{|c|}{ SPI (No. of genes present in $>=80 \%$ of isolates) ${ }^{a}$} \\
\hline & & & $\frac{\mathbb{3}}{5}$ & 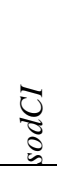 & 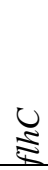 & 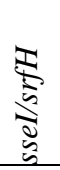 & 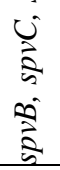 & $\begin{array}{l}\frac{0}{2} \\
\frac{\pi}{2} \\
2\end{array}$ & 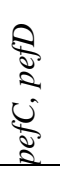 & 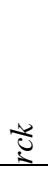 & 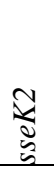 & $\begin{array}{l}\infty \\
\infty \\
0 \\
0\end{array}$ & $\underset{⿱ 亠 乂}{\vdots}$ & 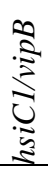 & 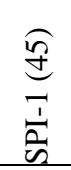 & 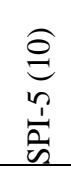 & 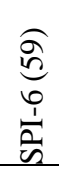 & $\begin{array}{l}\underset{a}{ \pm} \\
\frac{1}{a}\end{array}$ & 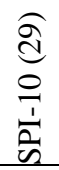 & 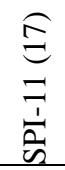 & $\begin{array}{l}0 \\
\frac{1}{2} \\
\frac{1}{n}\end{array}$ & $\begin{array}{l}\widehat{\vec{c}} \\
\stackrel{a}{1} \\
\frac{1}{\sqrt{n}}\end{array}$ & $\begin{array}{l}\underset{\sigma}{\sigma} \\
\underset{\sim}{\sim} \\
\frac{1}{2} \\
\tilde{n}\end{array}$ & 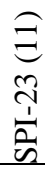 \\
\hline & A & 47 & + & & & & & & & & & & & & 42 & $10^{b}$ & 35 & 10 & 9 & 12 & 1 & $31^{b}$ & 2 & 5 \\
\hline MGT1-ST180 & $\mathrm{A}$ & 37 & + & & & & & & & & & & & & 42 & $10^{b}$ & 35 & 10 & 9 & 12 & 1 & $31^{b}$ & 2 & 2 \\
\hline MGT1-ST1972 & $\mathrm{C}$ & 33 & & + & + & & & & & & & & + & & $45^{b}$ & $10^{b}$ & 37 & 8 & 10 & 13 & $6^{b}$ & 30 & 2 & 6 \\
\hline MGT1-ST183 & $\mathrm{B}$ & 410 & & + & + & + & + & & & & & + & + & + & 42 & 8 & 35 & 44 & 10 & 13 & $6^{b}$ & $31^{b}$ & 2 & 5 \\
\hline MGT3-CC10 & $\mathrm{B}$ & 164 & & + & + & + & + & & & & & & & & 42 & $10^{b}$ & 19 & 10 & 10 & 13 & $6^{b}$ & 15 & 2 & 2 \\
\hline MGT3-CC15 & $\mathrm{B}$ & 274 & & + & + & + & + & + & + & + & & & & & 42 & $10^{b}$ & 19 & 49 & 10 & 13 & $6^{b}$ & 15 & 2 & 2 \\
\hline MGT3-CC107 & $\mathrm{B}$ & 364 & & + & + & + & + & + & & & + & & & & 42 & $10^{b}$ & 18 & 11 & 10 & 13 & $6^{b}$ & 15 & 2 & 3 \\
\hline MGT3-CC18 & B & 83 & & + & + & + & + & + & & + & + & & & & 42 & $10^{b}$ & 18 & 11 & 10 & 13 & $6^{b}$ & 14 & 2 & 2 \\
\hline MGT4-CC101 & $\mathrm{B}$ & 102 & & + & + & + & + & + & + & + & & & & & 42 & 9 & 19 & 11 & 10 & $17^{b}$ & $6^{b}$ & 15 & 2 & 5 \\
\hline MGT4-CC129 & B & 199 & + & + & + & + & + & + & + & + & + & + & & & 42 & $10^{b}$ & 19 & 10 & 10 & 13 & $6^{b}$ & 15 & 2 & 5 \\
\hline MGT4-CC30 & B & 135 & & + & + & + & + & + & + & + & & + & & & 42 & $10^{b}$ & 19 & 10 & 10 & 13 & $6^{b}$ & 15 & 2 & 5 \\
\hline MGT4-CC1 & B & 8539 & & + & + & + & + & + & + & + & + & & & & 42 & $10^{b}$ & 19 & 11 & 9 & 13 & $6^{b}$ & 15 & 2 & 5 \\
\hline MGT4-CC13 & $\mathrm{B}$ & 14927 & & + & + & + & + & + & + & + & & & & & 42 & $10^{b}$ & 19 & 45 & 10 & 13 & $6^{b}$ & 15 & 2 & 5 \\
\hline
\end{tabular}

2

$3{ }^{a}$ SPI-2, 3, 4, 9,12,13,14 and 16 were found intact in all of the STs/CCs; SPI-8, 15, 18, 20 and 21 were absent in all STs/CCs.

$4{ }^{b}$ The SPI was predicted to be intact in the ST/CC with all genes observed. 


\section{Figure legends:}

6 Fig. 1. Makeup and assignments of each $S$. Enteritidis MGT level. a. Number of loci included in

7 each MGT level. The first eight levels are composed of Salmonella enterica core genes, which are orthologous to those of the $S$. Typhimurium MGT1 to MGT8, except for one gene at MGT8 which was excluded due to duplication in $S$. Enteritidis. The MGT9 scheme includes core genes of S. Enteritidis (those not belonging to the Salmonella core coloured in yellow) and core intergenic 11 regions (coloured in green). The number behind or within each bar refers to the number of loci

12 included. b. The number of sequence types (ST) and clonal complexes (CC) assigned at each MGT 13 level among the 26,670 genomes analysed. CC includes STs of no more than one allele difference. 14 The numbers refer to the number of STs or CCs types assigned at each level, which were also indicated by colour. As MGT9 included 4986 loci with highest subtyping resolution, the 26,670 isolates were subtyped into 20,153 different ST types and 14,441 CC clustering types at MGT9.

Fig. 2. MGT4 STs offer a useful description of continent specific and global clades. a. At each MGT level from MGT2 to MGT8, we identified continent restricted STs where more than 70\% of the isolates belonged to the same continent. MGT4 was found to have the highest number of isolates belonged to these continental limited STs. b. There were 45 MGT4-STs of more than 50 isolates representing $73.5 \%$ of the total 26670 isolates, and the majority of these STs belonged to MGT4$\mathrm{CC} 1$ and CC13. The number, size and continental makeup of these STs is shown with the left Y axis showing MGT4 STs, the right $\mathrm{Y}$ axis showing MGT4 CCs and the X axis showing number of 25 isolates, continental distribution of each ST is shown by different colours in each row. 
30 ST25 and ST13 were prevalent in the UK, while MGT4-ST15 and ST25 were mixed. b. MGT4-

31 ST15 and ST25 can be subtyped into multiple STs at MGT5, the majority of which were prevalent in

32 the UK, except for MGT5-ST412 and ST387 in the USA.

Fig. 4. MGT4 ST proportions across states in the USA is similar but only a subset of STs are associated with avian hosts. For the USA $S$. Enteritidis isolates with metadata (4,383 isolates), a total of 3935 genomes were from either avian or human source, 95.0\% (3738/3935) of which belonged to MGT4-CC1 and CC13. The MGT4-STs belonged MGT4-CC1 and CC13 of either avian or human source were shown in different states of the USA. Each pie chart illustrated the MGT-ST types and the size of the main STs represented with different colours. The size of the pie indicated the total number of isolates in each state. While CC13 appears to originate in birds before moving to humans, the reservoir of $\mathrm{CC} 1$ is unknown. Origins for $\mathrm{CC} 1 \mathrm{STs}$ were also not observed in any other source type. The maps were created with Tableau v2019.2.

Fig. 5. MGT5 STs allow identification of temporal patterns in strain diversity in the UK. There were 13 MGT5-STs of more than 100 isolates shown with different colours. Temporal patterns indicate that some STs are endemic while others likely cause outbreaks. MGT5-ST156 in red was observed predominantly from June to August of 2014, and disappeared after 2014. MGT5-ST423 was a dominant type from March to September of 2015, and became dominant again during the September to December of 2016.

Fig. 6. Global phylogenetic structure of $S$. Enteritidis. A total of 1508 genomes were randomly

52 sampled from each ST in MGT6. A phylogenetic tree was constructed by ParSNP v1.2, which called 53 core-genome SNPs extracted from alleles. The tree scales indicated the 0.01 substitutions per locus. 54 The number on internal branches represented the percentage of bootstrap support. a. Three clades 
55 were identified which were concordant with previous findings: The predominant clade, highlighted

56 in yellow, is the global epidemic clade which was phylogenetically closer to $S$. Gallinarum than the

57 other two clades in purple and green background which are limited in Oceania 9. b. The main,

58 yellow highlighted clade was expanded into a more detailed phylogeny with $S$. Gallinarium as an

59 outgroup. 10 main lineages were defined where each lineage had more than 10 isolates. STs of the

60 seven gene MLST (or MGT1) and CCs from MGT2 to MGT4 are shown in different colours for each

61 of the 10 lineages. The pie charts for each lineage represent the proportion of the isolates belonged to

62 the same MGT4-CC shown on the right side.

63

64 Fig. 7. Skyline population size estimation of MGT4-CC1 and CC13. Bayesian skyline model and

65 strict clock were estimated to be the optimal combination. The vertical axis column indicates the

66 predicted log scale effective population size and the horizontal axis shows the predicted time. The

67 blue line represents the median posterior estimate of the effective population size, and the blue area

68 shows the upper and lower bounds of the 95\% highest posterior density interval. a. Skyline

69 population expansion of MGT4-CC1. Around 1950 and 1970, there were two large population

70 expansions within MGT4-CC1. b. Skyline population expansion of MGT4-CC13. The population of

71 MGT4-CC13 gradually expanding until around 1970, when there was also an accelerated expansion.

72

73 
bioRxiv preprint doi: https://doi.org/10.1101/2020.06.30.169953; this version posted April 22, 2021. The copyright holder for this preprint (which was not certified by peer review) is the author/funder. All rights reserved. No reuse allowed without permission.

a

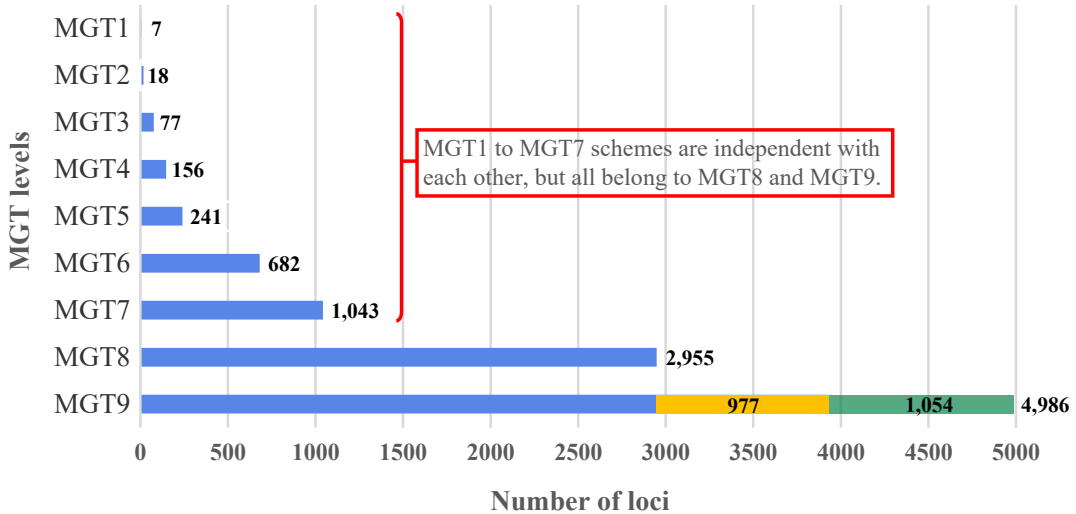

b MGT No. of STs No. of CCs

$\begin{array}{lll}\text { MGT2 } & 253 & 23\end{array}$

$\begin{array}{lll}\text { MGT3 } & 1,222 & 179\end{array}$

MGT4 2,236 423

MGT5 4,128 1,017

MGT6 7,262 2,838

MGT7 $\quad 11,107 \quad 5,680$

MGT8 $\quad 17,036 \quad 11,223$

MGT9 20,153 14,441

No. of types

$23 \quad 20,153$

$\because$ Salmonella species core genes $\backsim S$. Eneteritidis core genes $\square$. Eneteritidis core intergenic regions 
bioRxiv preprint doi: https://doi.org/10.1101/2020.06.30.169953; this version posted April 22, 2021. The copyright holder for this preprint (which $\mathbf{a}$ was not certified by peer review) is the author/funder. All rights reserved. No reuse allowed without permission.

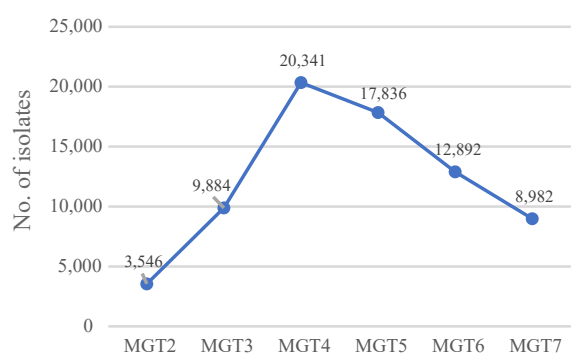

Continents

North America

Europe

South America

Asia

Oceania

Africa

None b

No. of isolates

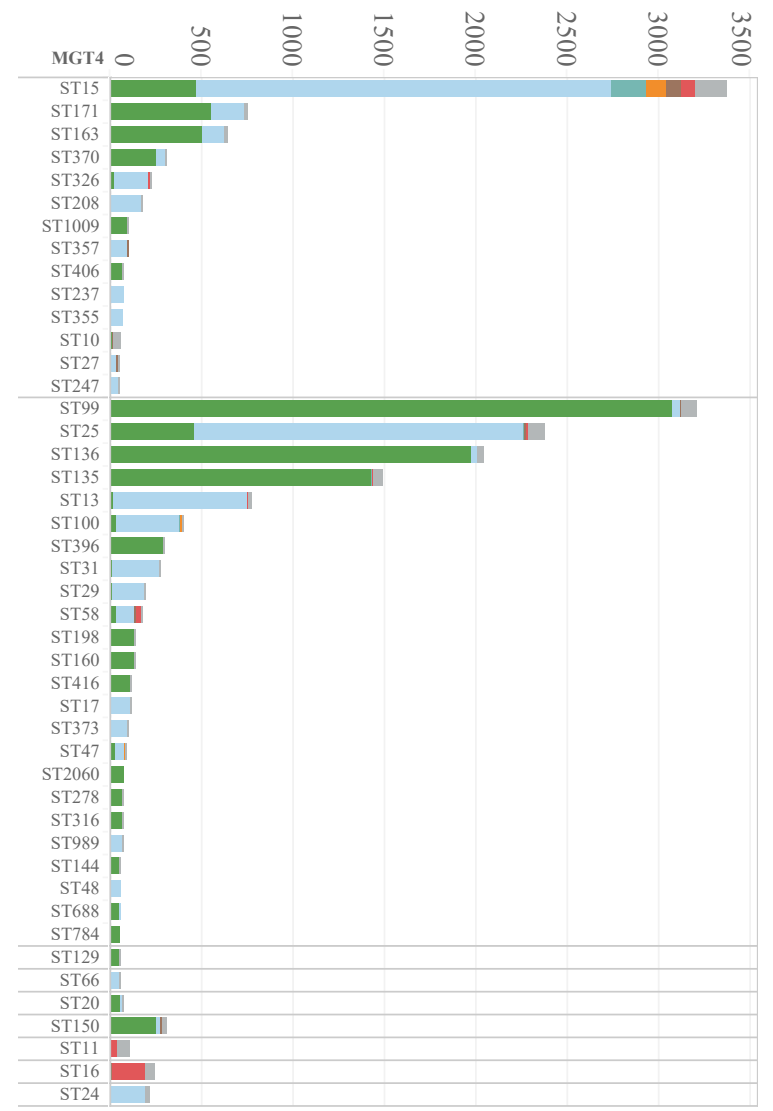

MGT4 MGT3 MGT2 MGT1

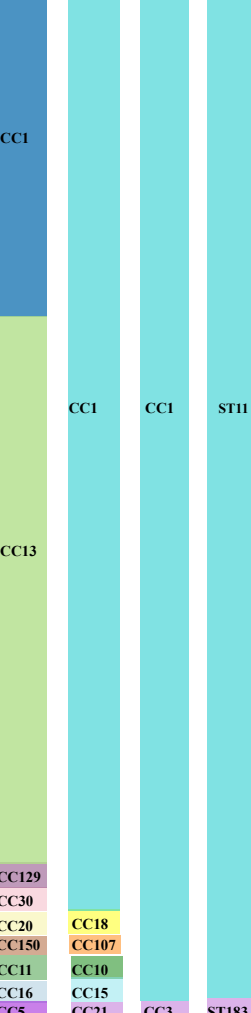


bioRxiv preprint doi: https://doi.org/10.1101/2020.06.30.169953; this version posted April 22, 2021. The copyright holder for this preprint (which wẩ not certified by peer review) is the author/funder. All rights reserved. No reuse allowed without permission.

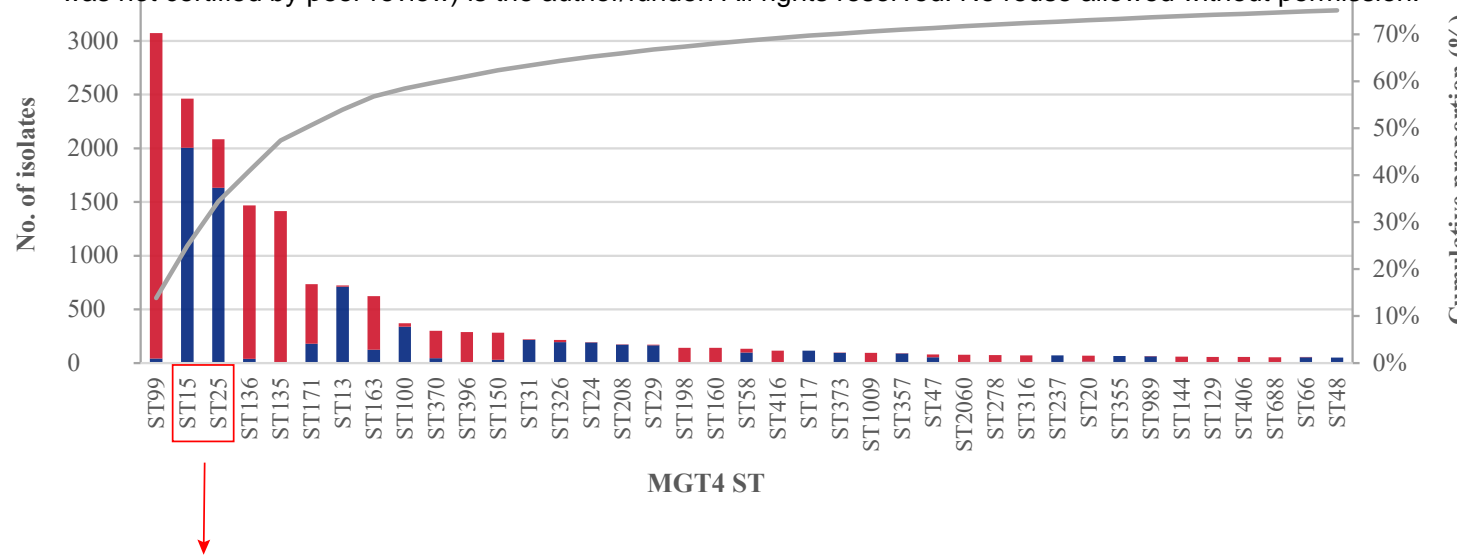

b

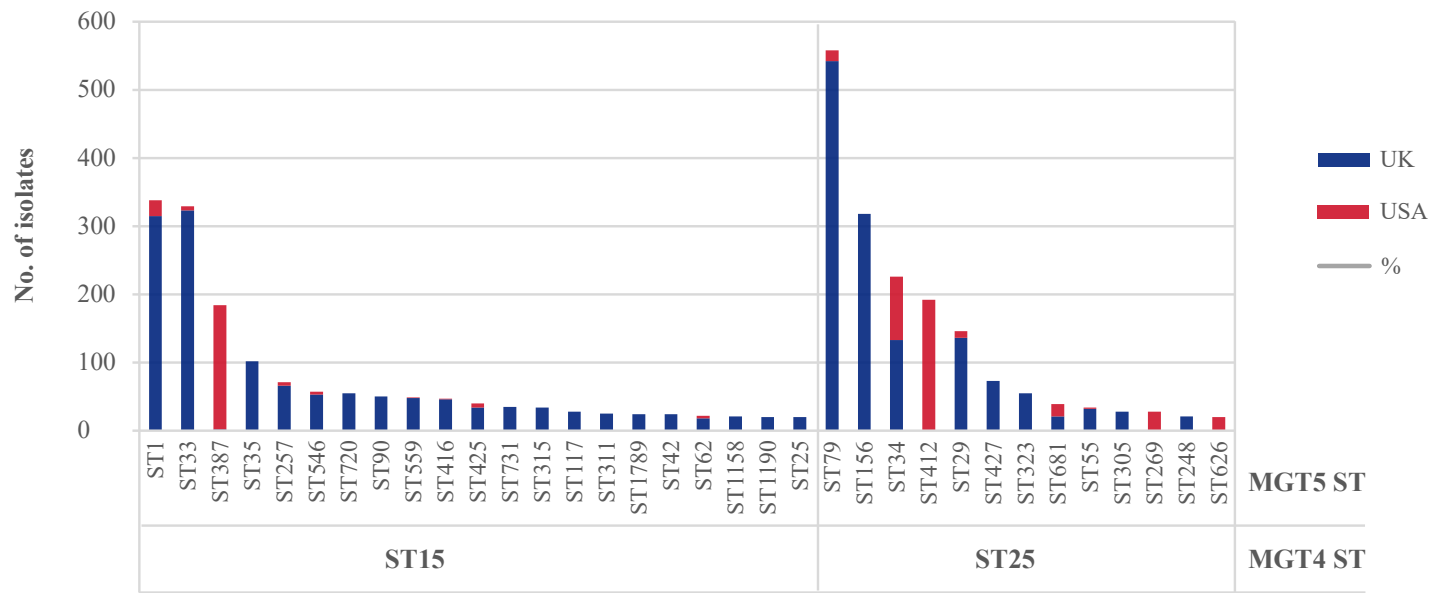


bioRxiv preprint doi: https://doi.org/10.1101/2020.06.30.169953; this version posted April 22, 2021. The copyright holder for this preprint (which

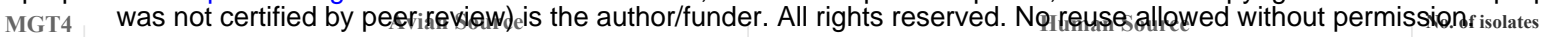

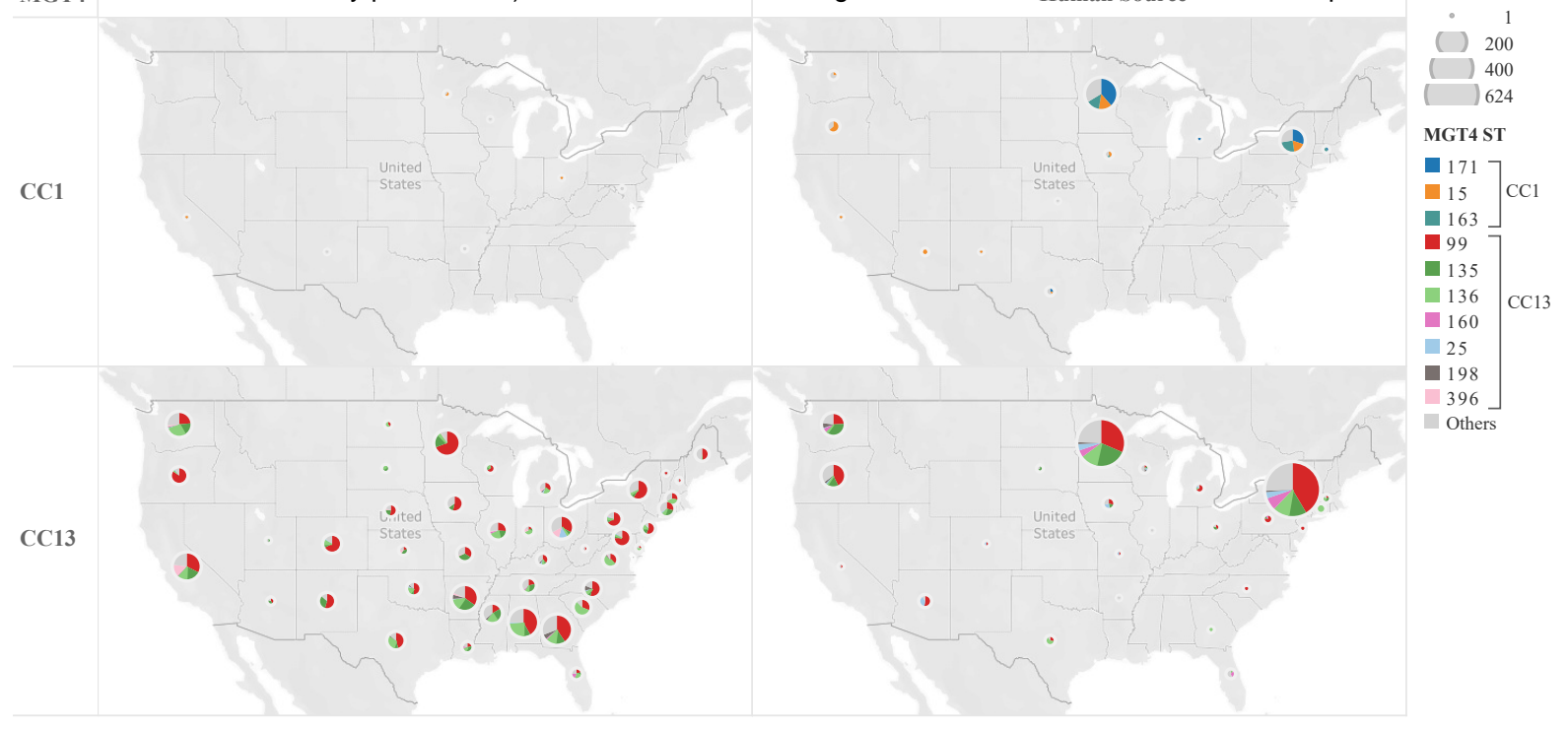




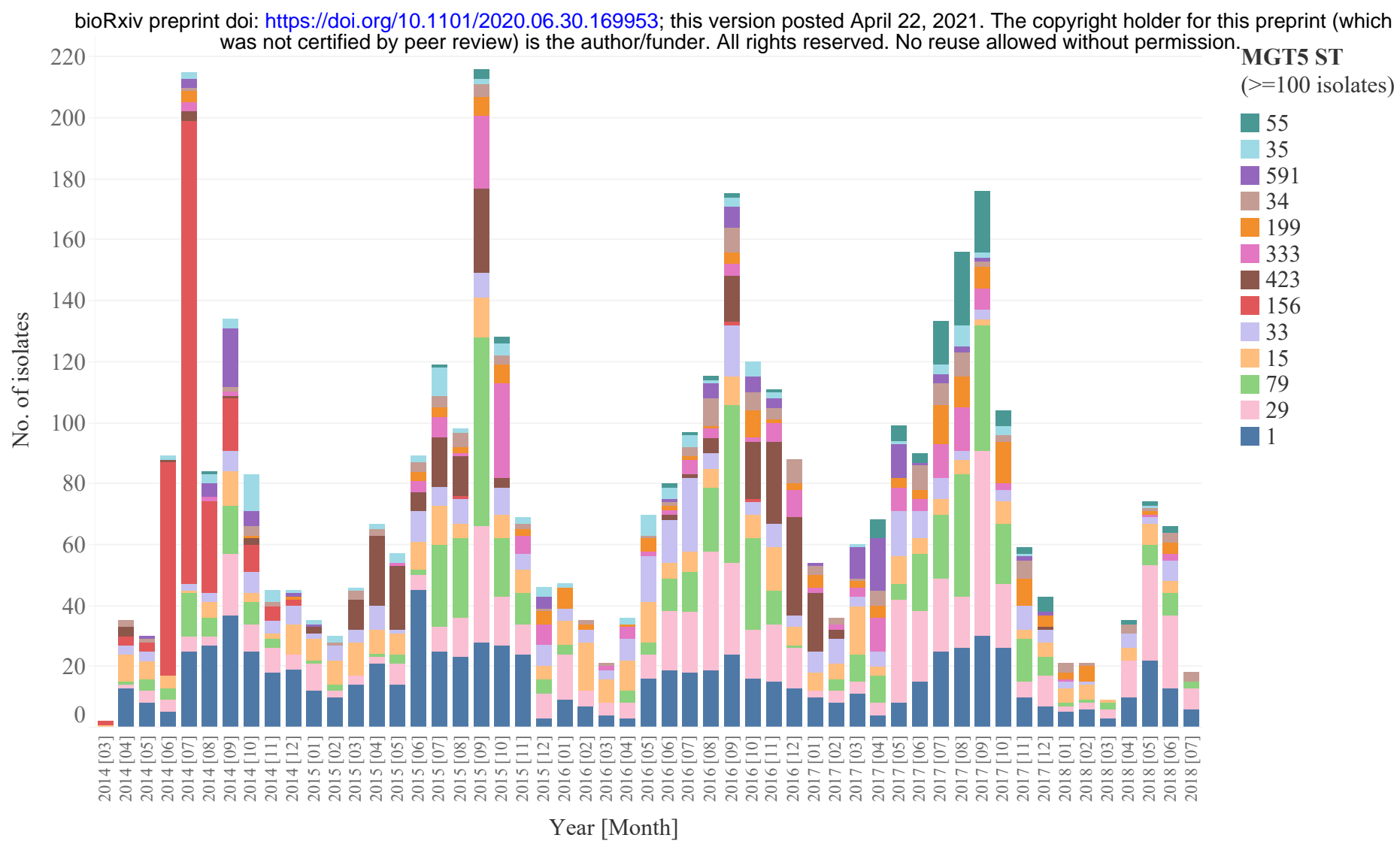


bioRxiv prep;int doi: https://doi.org/10.1101/2020.06.30.169953; this version posted April 22 2021. The copyright holder for this preprint (which was not certifiep by peer review) is the author/funder. All rights reserved. No teuse allowed without permission.

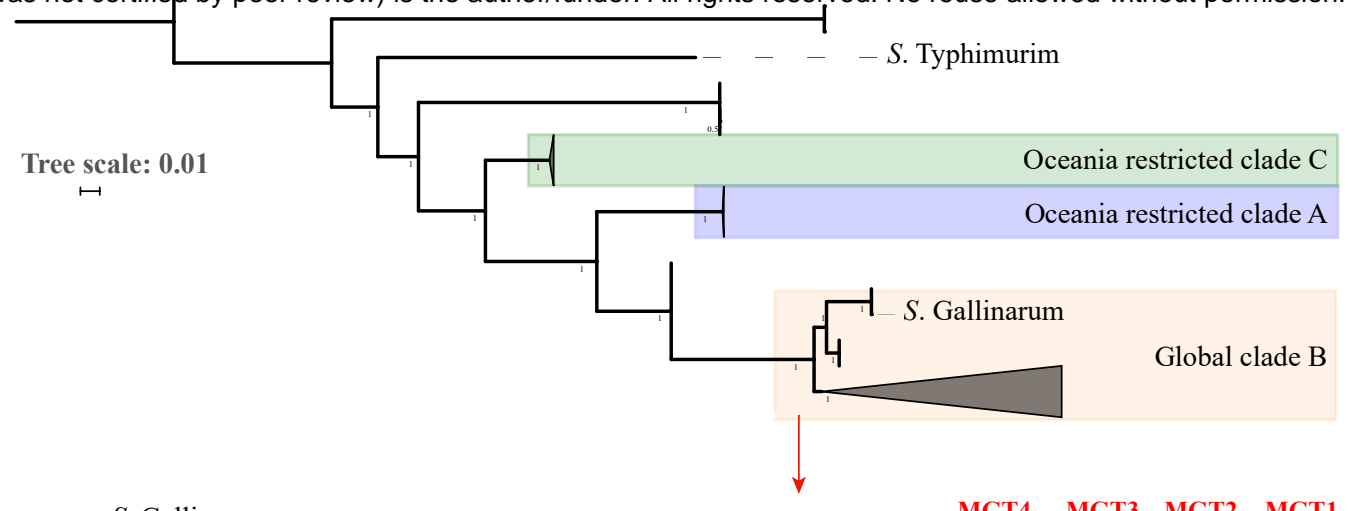

b

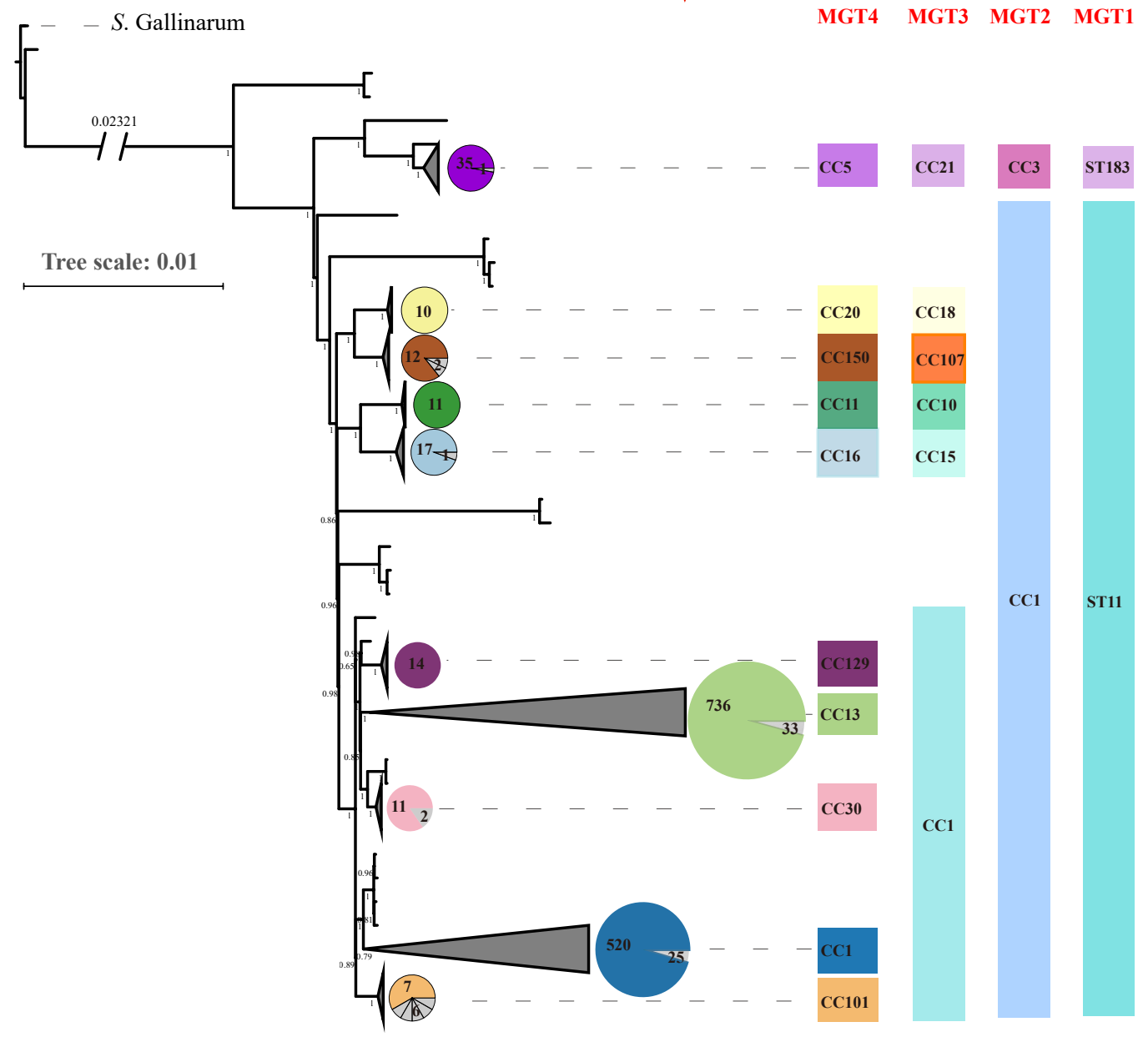


bioRrvopreprint doi: https://doi.org/10.1101/2020.06.30.169953; this version posted April 22, 2021. The copyright holder for this preprint fwhich

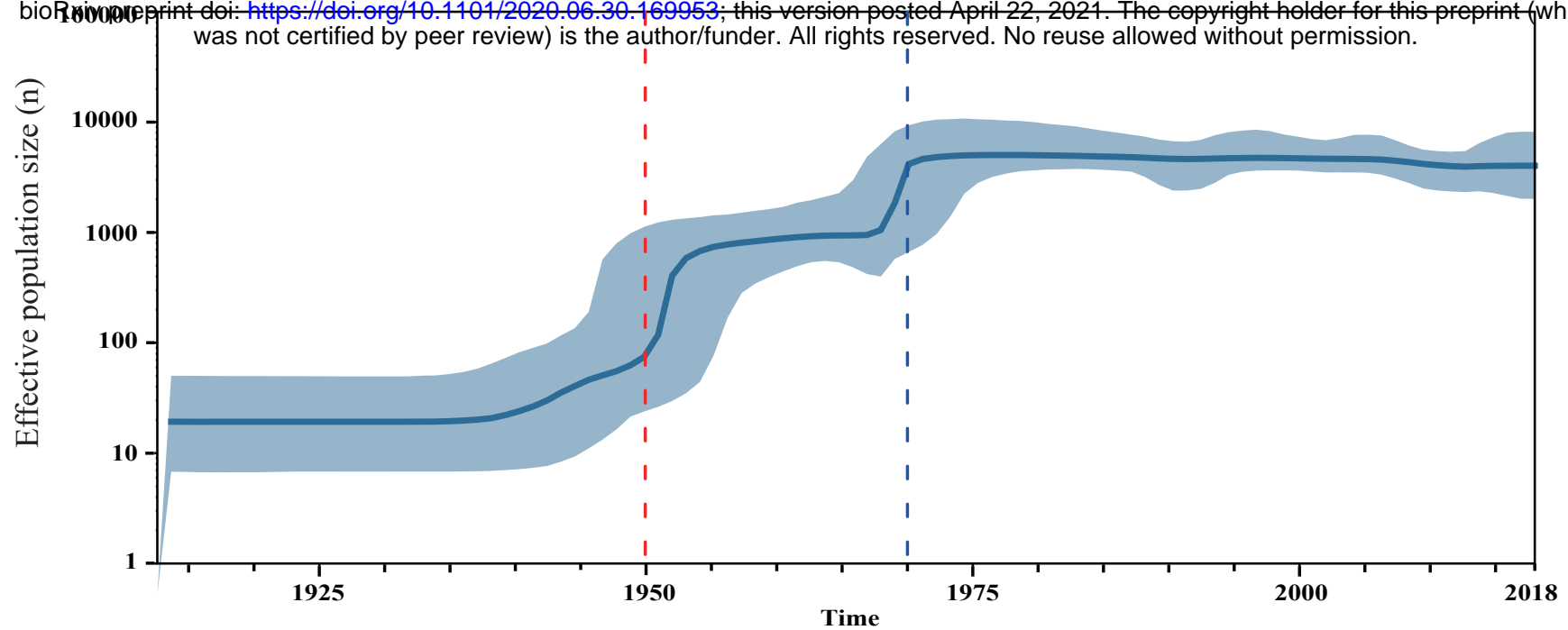

b

MGT4 CC13

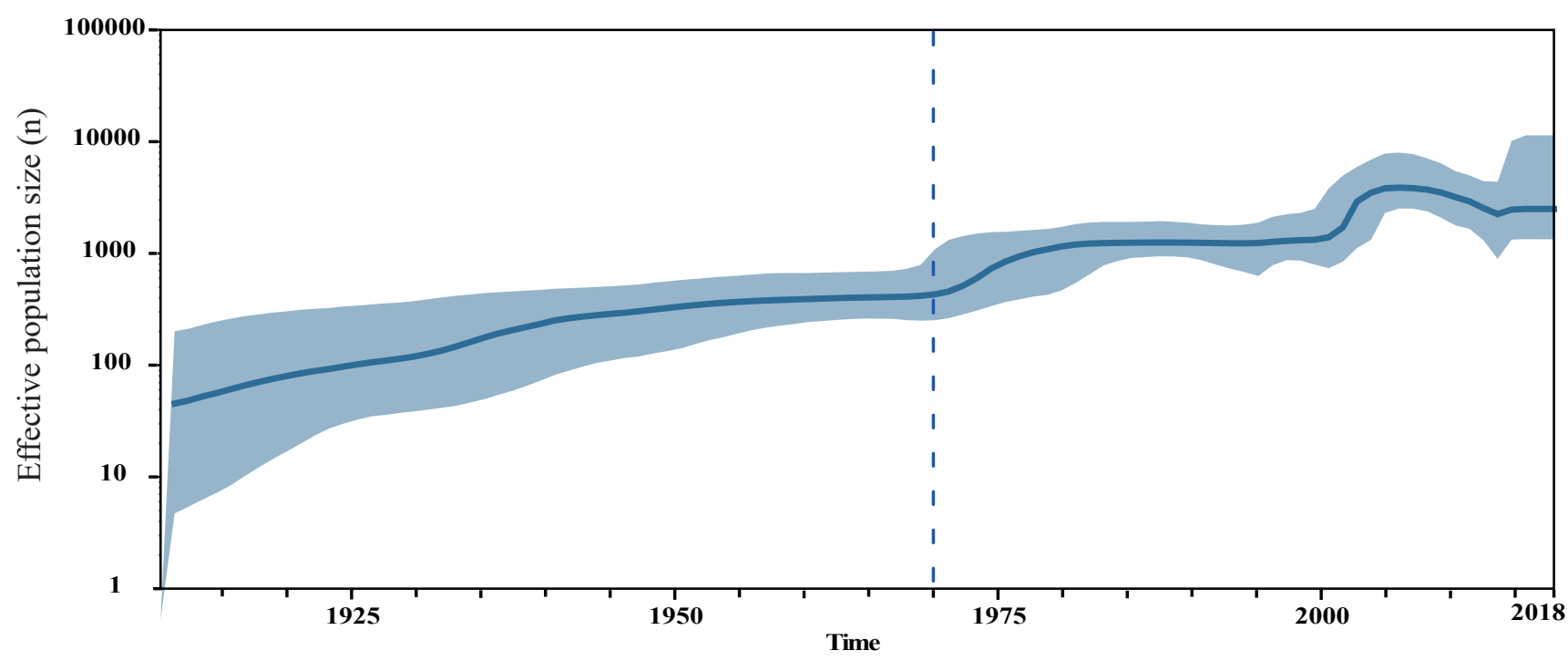

